\title{
Metodología para la predicción de puntos de riesgos múltiples en infraestructuras viarias tras episodios torrenciales (road-risk)
}

Methodology for the prediction of multiple risks points in road infrastructures after torrential episodes (road-risk)

\author{
María Jesús Perles Roselló \\ mjperles@uma.es \\ Departamento de Geografía \\ Universidad de Málaga (España)
}

\section{Santiago Manuel Pardo García \\ pardo@uma.es}

Grupo de Investigación Análisis Geográfico

Universidad de Málaga (España)

\section{Matías Mérida Rodríguez}

mmerida@uma.es

Departamento de Análisis Geográfico Regional y Geografía Física

Universidad de Málaga (España)

Jorge Olcina Cantos

jorge.olcina@ua.es

Departamento de Geografía

Universidad de Alicante (España) 


\title{
Resumen
}

Se presentan los contenidos de una metodología que permite predecir y cartografiar aquellos puntos en el recorrido de una infraestructura viaria susceptibles de quedar bloqueados por riesgos tras episodios de precipitación torrencial. Se consideran los riesgos de movimiento en masa, subsidencia del firme, generación de balsas e inundación por desbordamiento de cauces aledaños. Para estimar las consecuencias en cada punto, se evalúan tanto factores de peligrosidad como de vulnerabilidad del medio humano frente al potencial corte de tráfico. Se ha diseñado, además, una aplicación informática que implementa la metodología obtenida y facilita su aplicación a otros casos.

Palabras clave: multi-riesgos; infraestructuras viarias críticas; movimientos en masa; inundación; vulnerabilidad frente al riesgo.

\begin{abstract}
The paper contains an applied methodology that allows mapping the points in the road that can be blocked by multiple risks of simultaneous operation, after an episode of high intensity rains. It incorporates two predictive models to identify points where there are mass movement risks, subsidence under the infrastructure and/or waterlogging. Moreover, it has been designed a software application that applies the obtained prediction criteria and maps the conflicted points in infrastructures from a different study area.
\end{abstract}

Key words: multiple risks; road critical infrastructures; landslides; waterlogging; rainfall.

\section{Introducción}

Las infraestructuras viarias son elementos del territorio de carácter estratégico para el funcionamiento de los servicios territoriales básicos, por lo que adquieren especial trascendencia en momentos de crisis. Las precipitaciones de alta intensidad horaria, características del clima mediterráneo, actúan como detonante de un conjunto de riesgos de origen diverso cuya gestión ha sido abordada tradicionalmente como problemas independientes. Sin embargo, vertebrados por la infraestructura, estos riesgos tienen en común que confluyen en el espacio y en el tiempo, y su prevención requiere de soluciones conjuntas e interconectadas (Perles \& Cantarero, 2010). Por este motivo, desde nuestra perspectiva, es necesario un cambio en la gestión de los mismos desde un enfoque sectorial, a uno transversal e integrado, objetivo para el que previamente, es preciso habilitar metodologías de análisis del problema ad hoc.

El entorno de las infraestructuras viarias posee unas características morfológicas y funcionales que las definen como espacios de riesgo particulares, especialmente susceptibles a ser afectados por la 
peligrosidad, además de particularmente vulnerables ${ }^{1}$. Requieren un tratamiento singularizado del riesgo por su naturaleza compleja, y pueden considerarse, por las características que se detallan a continuación, unidades territoriales de riesgo específicas, en el sentido en que son definidas por Calvo (2001) o Perles y Mérida (2010).

Desde el punto de vista de la peligrosidad, en estos espacios los procesos hidro-geomorfológicos tienen que adaptar su dinámica a un entorno de transición brusca entre lo natural y lo artificial (Luce \& Wemple, 2001; Perles \& Cantarero, 2010; Corominas et al., 2013). Los flujos de escorrentía, interrumpidos por la propia infraestructura, han de ajustarse a condiciones artificiales de aceleración o remanso a través de culverts transversales, conducciones longitudinales, bermas, cunetas, etc. Los flujos hídricos y gravitacionales han de adaptarse igualmente a unas condiciones de pendiente alteradas por los desmontes, taludes, terraplenes, rellenos etc., que modifican la estabilidad de laderas. El resultado de todas estas alteraciones se concreta en problemas de peligrosidad que son propios y específicos de las infraestructuras viarias, tales como balsas con riesgo de aquaplaning, invasión de la carretera por corrientes rápidas con riesgo de arrastre de vehículos, hundimientos del firme e incluso destrucción de puentes. A la vez, la energía cinética del evento torrencial puede activar movimientos en masa en las laderas adyacentes a la infraestructura, que, a su vez, provocan bloqueos de distinta severidad en la calzada, o subsidencia del firme si la inestabilización se produce en la ladera subyacente a la calzada.

Desde el punto de vista de la vulnerabilidad, también la infraestructura constituye un espacio singular de riesgo, ya que el tránsito viario implica una exposición directa de la vida humana (la mayor parte de los siniestros y fallecimientos durante los episodios de lluvias torrenciales se producen en carreteras). Por otra parte, el tráfico es un factor estratégico para el normal funcionamiento de los servicios territoriales, por lo que su interrupción tiene impactos múltiples sobre la población y actividades del entorno. Conocer de antemano dónde se producirá el punto problemático de bloqueo permite poner en marcha labores preventivas en los puntos más peligrosos. Si además se analiza el tipo de servicio que presta la carretera en cada tramo,

1 Ecuación General del Riesgo: La UNDRO (United Nations Disaster Relief Organization) define el riesgo como el producto de la peligrosidad originada por una amenaza, por el volumen de elementos expuestos ponderados por su vulnerabilidad (Riesgo= Peligrosidad Exposición* Vulnerabilidad).

Riesgo: Probabilidad de que un elemento expuesto sufra un daño como consecuencia de la acción de un peligro.

Peligro: proceso o fenómeno de carácter natural o tecnológico que puede originar daños a la población, los bienes materiales o al medio ambiente natural (Olcina \& Ayala, 2002)

Exposición: conjunto de bienes a preservar que pueden ser dañados por la acción de un peligro (Olcina \& Ayala, 2002). La Directiva 2007/60/CE sobre el riesgo de inundación especifica como bienes a preservar la salud y vida humana, el medio ambiente, el patrimonio cultural, la actividad económica y las infraestructuras.

Vulnerabilidad: características de una persona o grupo de personas en términos de su capacidad para anticipar, gestionar, resistir o recuperarse del impacto de un riesgo (Blaikie et al., 1994). 
podremos dar prioridad en la prevención a aquellos puntos de la infraestructura viaria en los que el bloqueo del tráfico afecte a un mayor número de personas, y/ o a funciones del territorio de mayor trascendencia y vulnerabilidad

Por las particularidades de la producción del riesgo en las infraestructuras viarias, se observa la necesidad de idear metodologías multi-peligrosidad y multi-riesgo para el tratamiento unitario del problema en estos espacios, métodos de enfoque holístico frente al sectorial, que integren peligros de distinto origen, causas naturales y/o artificiales y factores de peligrosidad, junto a los de vulnerabilidad. Se detecta un vacío en este tipo de metodologías, ya que los enfoques integrales han sido tratados en literatura más de una forma teórica que aplicada (Cutter, 1994), y en otras ocasiones han sido enfocados desde una escala de trabajo más divulgativa que aplicable (Greiving, Fleischauer \& Lückenköter, 2004). Junto a este vacío, diversos autores apuntan el actual contexto de oportunidad para las metodologías predictivas de apoyo a la prevención de desastres en las consideradas "infraestructuras críticas" (Zhao, Li \& Fang, 2018), metodologías que están adquiriendo una especial significación y trascendencia en el contexto de la promulgación de leyes específicas para la Protección de Infraestructuras Críticas, tanto a nivel europeo (2008/114 CE), como español (Ley 8/2011), y de la creación del Centro Nacional para la Protección de Infraestructuras Críticas. De igual modo, trabajos recientes indican la necesidad de este tipo de enfoques ante el previsible incremento de los riesgos en las infraestructuras críticas en un escenario de cambio climático (Rsavdaroglou et al., 2018; Petrucci \& Pasqua, 2012; Cutter et al., 2018).

En este contexto de vacío, y, a la vez, de oportunidad, se propone una metodología fundamentada en un planteamiento fenomenológico, holístico y finalista, orientado hacia el trabajo en escalas medias, y aplicable a la resolución de problemas a nivel territorial en estos espacios estratégicos del territorio.

En el artículo se aporta, en primer lugar, una explicación de los antecedentes y bases conceptuales en la que se fundamenta el planteamiento metodológico, y su grado de innovación. A continuación se expone la metodología propuesta, que se articula en torno a dos bloques: el Bloque I, referente a la peligrosidad de bloqueo del tráfico por movimientos en masa y/o inundación de la calzada, y el Bloque II, que se centra en una estimación de la exposición y vulnerabilidad de la infraestructura viaria a partir de las alteraciones de la accesibilidad y de las afecciones sobre la funcionalidad del territorio producidas por el posible bloqueo del tráfico. Tras abordar a continuación la estrategia de unificación de la peligrosidad y la vulnerabilidad, para la obtención final de los puntos conflictivos de riesgo potenciales, se explican, en último lugar, las características básicas de la aplicación informática generada para implementar y aplicar la metodología, de forma ágil, a cualquier otro punto del territorio. 


\section{Objetivos}

El objetivo final del artículo es mostrar la estructura general y fundamentos de una propuesta metodológica implementada en una aplicación informática que permite identificar y detectar en el espacio aquellos puntos en el recorrido de una infraestructura viaria que, por sus características de peligrosidad y vulnerabilidad, puedan constituir puntos de riesgo especialmente conflictivos en episodios de precipitaciones de alta intensidad. De este modo, de forma más específica, en el trabajo se abordan los siguientes objetivos:

- Identificar los problemas de riesgo que se producen en las infraestructuras viarias tras episodios de lluvias torrenciales, y analizar sus factores causantes. Caracterizar el funcionamiento particular de los procesos de peligrosidad al adaptarse al entorno antropizado de la infraestructura.

- Deducir un modelo predictivo de la probabilidad de bloqueo de la carretera en cada punto a causa de peligros múltiples de funcionamiento asociado tras episodios torrenciales (peligros asociados). Se han analizado esencialmente diversos eventos peligrosos relacionados con movimientos gravitacionales y con la inundación de la calzada.

- Diseñar una estrategia metodológica para evaluar la magnitud de población, bienes y servicios afectables por el cierre del tránsito viario en el punto conflictivo identificado (exposición y vulnerabilidad frente al peligro).

- Generar una interfaz para la aplicación de la metodología diseñada a otras infraestructuras, tanto en fase de funcionamiento como de proyecto.

\section{3 Áreas de estudio}

Las áreas de estudio utilizadas para diseñar el modelo predictivo y mostrar los resultados de su aplicación (ver Figura 1) se corresponden con distintos tramos de carreteras andaluzas (A-357 de Ronda a Málaga, A-7 Circunvalación de Málaga y A-44 de Motril a Dúrcal), aunque en este trabajo se muestra únicamente una selección de mapas ilustrativos. Se ha procurado seleccionar zonas con peligrosidad potencial elevada (alta pendiente, litologías susceptibles a la movilización, elevados coeficientes de escorrentía, etc.). De igual modo, se ha considerado como criterio de selección la funcionalidad del territorio al que la carretera en cuestión da servicio, para recoger entre las zonas piloto una casuística de modelos territoriales funcionalmente diversos, que a su vez definirán distintos escenarios de vulnerabilidad (según tipo de poblamiento, accesibilidad, actividad económica principal del territorio, etc.).

Una revisión a la casuística en el área de estudio muestra que se trata de una zona con una problemática recurrente en la temática tratada, los episodios multi-riesgos con impactos sinérgicos sobre el tránsito viario (ver Figura 2). 
Figura 1. Tramos de carretera seleccionados como área de estudio

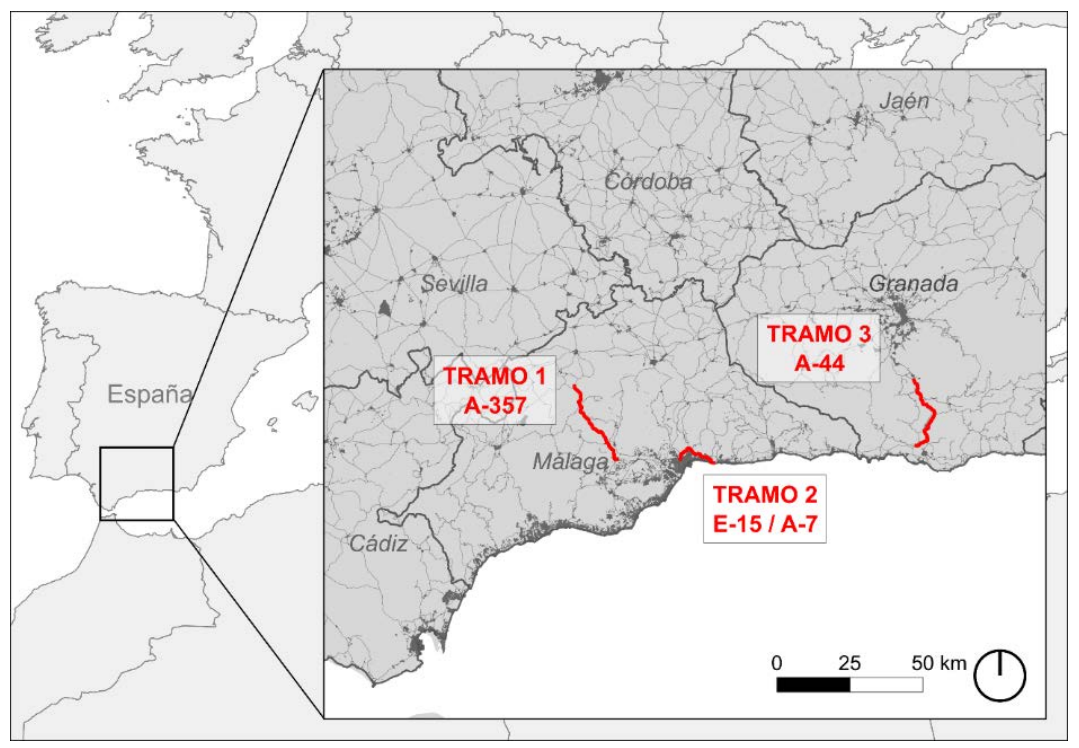

Fuente: elaboración propia

Figura 2. Hundimientos de calzada en Salares (Málaga) (a) y Málaga (b).

Balsa de agua en autovía A7 (Málaga) (c).
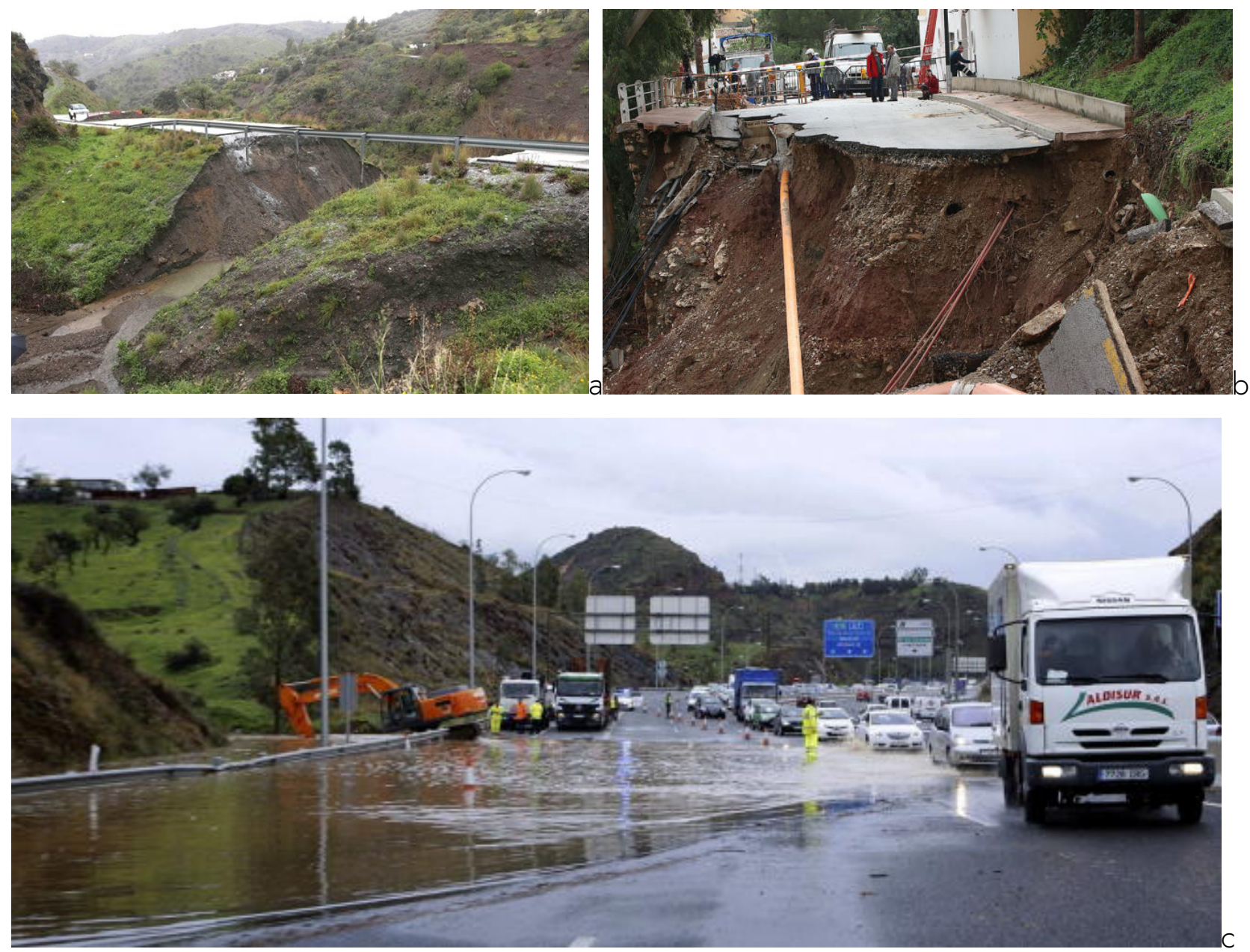

Fuentes: Europa Press (2018) (a) y La Opinión de Málaga (2017; 2014) (b y c) 


\section{Antecedentes y bases conceptuales de la propuesta metodológica}

\subsection{El riesgo como proceso territorial}

La propuesta metodológica se fundamenta en un enfoque de los problemas de riesgo entendidos como un hecho territorial. Desde esta perspectiva se postula que las características territoriales del espacio de riesgo a analizar condicionan el funcionamiento de los procesos de peligrosidad y vulnerabilidad, los singulariza, y, por ello, las metodologías para evaluar la generación del riesgo en determinados patrones territoriales deben adaptarse al espacio en el que se producen. Este enfoque subyace en el concepto de "lugar de riesgo" de Cutter (Cutter, 1994; Cutter, Boruff \& Shirley, 2003), y otras nociones similares aportadas por Calvo (2001), Olcina (2008), Perles y Mérida (2010) o Veyret, Beucher y Bonnard (2005), que propugnan el tránsito desde el concepto de riesgos naturales hacia el de territoriales. Esta perspectiva, según apuntan (Perles y Mérida, 2010), implica una sustitución del enfoque sectorial (análisis de riesgos individuales desde orientaciones disciplinares y a partir de unidades naturales), por un planteamiento fenomenológico, fundamentado en la observación de la realidad de forma integrada, tal y como se manifiesta en el territorio. El objeto de análisis se centra, por tanto, en los distintos espacios diferenciados en los que confluyen problemas multi-riesgo y en sus singularidades. Los sectores del territorio individualizados por su comportamiento homogéneo frente al riesgo constituyen, desde esta perspectiva, la unidad de análisis del riesgo. Se trata, además, de espacios gestionados admistrativamente por un organismo que los regula y del que depende preferentemente la solución y mitigación de los problemas de riesgo.

\subsection{La infraestructura viaria como espacio de singular de riesgo (unidad territorial de respuesta homogénea frente al riesgo)}

Como se detalla a continuación, las infrastructuras viarias y su entorno, por sus características morfológicas y fisiográficas particulares, y por su singularidad funcional, constituyen un espacio de riesgo particularizado, que exige la adaptación de las metodologías de evaluación a su particular identidad. En lo que se refiere al funcionamiento de la peligrosidad, la infraestructura es un espacio receptor de riesgos de distinto origen (gravitacional, hídrico), que sin embargo pueden confluir en el tiempo, lo que propicia su funcionamiento sinérgico, y la necesidad de tratarlos como peligros asociados. Otra particularidad del entorno de las infraestructuras lineales como espacios de riesgo es la muy elevada intensidad de la inducción artificial de la peligrosidad. En tanto que transición o interfase entre lo natural y lo artificial, es un espacio complejo, en el que los procesos de peligrosidad pueden reaccionar de manera incierta y producir consecuencias no previstas. En lo que se refiere al componente de exposición y vulnerabilidad frente al riesgo, la infraestructura viaria es también un objeto territorial singular, ya que no sólo se expone al riesgo la propia construcción, sino el tráfico y el conjunto de servicios territoriales que dependen de él. Los impactos tiene 
externalidades sobre un entorno que excede con mucho el ámbito espacial de la carretera, y por ello, la evaluación de elementos expuestos y de su vulnerabilidad ha de adaptarse a este hecho. Otro factor que anima a tratar el espacio de las infraestructuras viarias como un caso de "lugar de riesgo" particular es el hecho de que posean su propio organismo de gestión, que actúa de forma transversal a las políticas sectoriales de riesgos, y que centraliza la aplicación de soluciones a los problemas que se producen sobre este espacio. En una propuesta metodológica aplicada, este hecho debe condicionar objetivos y procedimientos. Una metodología de evaluación del riesgo no es válida si los resultados que ofrece son incongruentes con los requerimientos (escala, información disponible) o competencias del organismo responsable de la gestión del riesgo en ese espacio. Se enmarca esta reflexión en la observación aportada por Clark (1998), que propone un cambio desde la fase de "metodologías para el análisis del riesgo" hacia la de de "métodos para la construcción de la seguridad". En definitiva, tanto el patrón territorial como los procesos asociados y las medidas de mitigación necesarias para la gestión del riesgo, singularizan cada una de estas unidades territoriales, y, en consecuencia, las metodologías de análisis y evaluación del riesgo deberían adaptarse a su especificidad.

\subsection{Tratamiento integral de los riesgos en la infraestructura viaria}

Existen antecedentes múltiples en lo que se refiere a cada uno de los tipos de riesgos tratados (trabajos sobre movimientos en masa o problemas inundación). La susceptibilidad del terreno a los movimientos en masa ha sido ampliamente estudiada cuando estos se producen en un entorno natural. Inicialment se han usado métodos geomorfológicos, y progresivamente se han incorporado al análisis estadísticos multivariantes y técnicas SIG (Guzzetti et al., 1999; Chacón et al., 2003; Ardizzone et al., 2002; Carrasco et al., 2003; González-jiménez et al., 2007; Fell et al., 2008; Felicisimo et al., 2013; Razavizadeh et al., 2017, entre otros). De igual modo, las aportaciones metodológicas y aplicaciones sobre riesgo de inundación, sólo en el ámbito español, son inumerables (Saurí \& Ribas, 1994 y 2004; Perles, 1999; Mayer, 2002; Ollero, 2014; Llorente, Diez \& Laín, 2006; Olcina, 2006; Camarasa, 2008; Olcina \& Diez, 2017). Lo más común ha sido analizar la incidencia de cada uno de estos peligros cómo procesos independientes, bajo la perspectiva sectorial de los especialistas en cada materia.

Sin embargo, por su particular casuística, son menos frecuentes los trabajos de susceptibilidad a los movimientos en masa o problemas de inundación específicamente referidos y adaptados al entorno de carreteras (Mérida, Perles \& Blanco, 1998; Chau et al., 2004; Remondo et al., 2005; Ayalew \& Yamagishi 2005; Youssef, Pradhan \& Hassan, 2011; Ataollah et al., 2012; Corominas et al., 2013; Delmonaco, Margottini \& Spizzino, 2006; San Millán et al., 2016, Sortino et al., 2016), y aún son menos comunes los trabajos referidos a la afección de carreteras por peligros múltiples de funcionamiento asociado. Pueden citarse análisis de las interaciones entre la erosión y la carga sólida de canales transversales a la carretera, o entre ésta y la estabilidad de taludes, y puentes 
(Simon \& Downs, 1995; Lagasse, Schall \& Richardson, 2001; Conesa \& García, 2013; 2014), aunque, en la mayoría de los casos estos trabajos se desarrollan como informes técnicos a nivel de proyecto de obra, con resultados muy precisos pero poco extrapolables a nivel del conjunto de una infraestructura (Brice, 1984, Furniss et al., 1998).

En lo que se refiere a la vulnerabilidad, existen antecedentes de trabajos sobre vulnerabilidad del medio humano frente a riesgos ambientales en el ámbito de la carretera, pero responden preferentemente a la estimación de pérdidas o afecciones sobre la propia calzada (Montz, 1994), y no se llegan a valorar los riesgos sobre la conducción y las repercusiones del corte de tráfico sobre la población y sus bienes. Son más escasas las aportaciones que abordan la vulnerabilidad de la infraestructura en razón de la alteración de la funcionalidad del territorio al que dan servicio (Perles \& Mérida, 2010; Youssef et al., 2011; Perles, Vías \& Andreo, 2008), y tampoco son comunes los trabajos que aplican una metodología que integre los aspectos naturales y humanos que generan el riesgo en este espacio particular (Lexer, Paluchova \& Schwarzl, 2008; Delmonaco et al., 2006).

La metodología que proponemos, se basa en el concepto de riesgos asociados (Casale \& Margotinni, 1999; Delmonaco et al., Perles \& Cantarero, 2010; Perles \& Mérida, 2010; Tibaldi, Ferrari \& Pasquare, 1995; Pedraza et al., 2004; Greiving, Fleischhauer \& Lückenkotter, 2004), que integra las repercusiones conjuntas que comportan riesgos de distinta etiología cuando actúan de forma simultánea en un mismo espacio. Algunos trabajos pueden citarse como ejemplo de un mayor grado de complejidad en este enfoque integral de los riesgos múltiples en el entorno de la carretera a escalas medias. La metodología propuesta por Younis (2004) avanza en la idea de cartografía predictiva multi-riesgo al proponer un índice para la optimización del mantenimiento de puentes en razón al peligro de fallo y al coste del potencial corte del tráfico que el corte pueda producir. Conesa, García y Pérez-Cutillas (2017) proponen estimar el riesgo en las infraestructuras integrando un indicador de peligro basado en parámetros hidráulicos, junto a la información sobre vulnerabilidad de la carretera basado en su categoría y en el trafico anual.

\subsection{El concepto de punto conflictivo o punto de riesgo}

A partir de los antecedentes y bases conceptuales citadas, la metodología predictiva que se propone en el trabajo pretende responder a las siguientes preguntas: ¿̇ué peligros se activan en el entorno de una infraestructura viaria tras un episodio torrencial? ¿En qué punto del trazado será más probable que se activen y bloqueen la carretera? Y, por último, ¿dónde las consecuencias de un bloqueo del tráfico serán más graves para la población y la funcionalidad del territorio?

Para responder a estas preguntas, partiendo de los conceptos generales de la Ecuación General del Riesgo $(R=P * V e)^{2}$, se ha definido la noción de punto conflictivo o punto de riesgo. Un punto

2 R=riesgo; P=peligro; $V e=$ vulnerabilidad de los elementos expuestos. 
conflictivo es aquel en el concurren una alta probabilidad de bloqueo del tráfico por peligros ambientales de su entorno, y que, a su vez, produzca una desconexión del territorio con consecuencias más graves para la población y su actividad. La conflictividad del punto, por tanto, es proporcional a la peligrosidad que le afecta potencialmente, y que define la probabilidad de que el punto pueda bloquearse; adicionalmente, el impacto más o menos grave del evento catastrófico dependerá de la intensidad del tráfico que circule por el punto en cuestión y de sus características. Según sea la localización del mismo, y su posición topológica en la red de infraestructuras, el bloqueo del tráfico tendrá consecuencias más o menos graves sobre los flujos de población y mercancías del entorno (Zhao, Li, Fang, 2018; Rsavdaroflou et al., 2018), flujos que a su vez serán más vulnerables si son más prioritarios o perentorios para el funcionamiento de los servicios territoriales básicos.

Figura 3. Esquema representativo de un punto conflictivo.

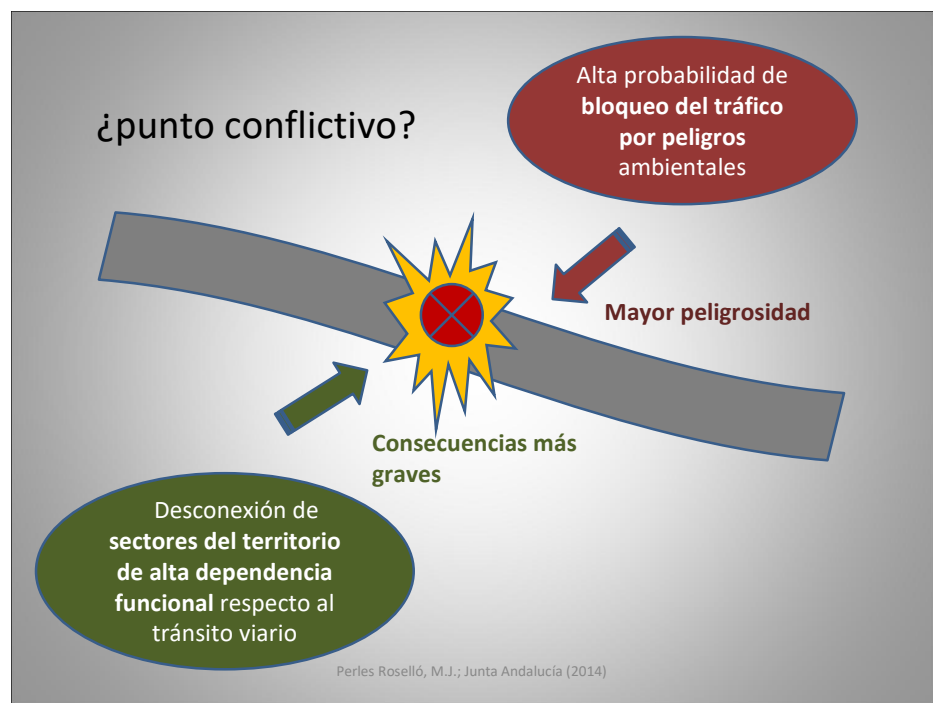

Fuente: elaboración propia

Para estimar las consecuencias de la peligrosidad sobre un espacio de riesgo específico como es la infraestructura viaria, la noción de punto conflictivo o punto de riesgo debe integrar los conceptos de peligrosidad múltiple y los de exposición socio-económica. Debe recoger, además, la idea de que la posición del punto peligroso en el entramado topológico de la red viaria actúa como condicionante de sus consecuencias efectivas, de su impacto, y debe considerar que, en este proceso de riesgo, existe una marcada transferencia espacial entre las áreas en las que se causa la peligrosidad, y las que reciben el impacto.

\subsection{Finalidad aplicada de la metodología. Operatividad de escala y fuentes}

El enfoque utilizado como fundamento de la metodología orienta la misma hacia una aplicación finalista, esto es, la determinación de cuáles serán los puntos de riesgo más conflictivos, y por tanto, a priorizar por la administración responsable de la gestión de la infraestructura a la hora de 
proponer o reforzar medidas de mitigación del riesgo en la infraestructura viaria. Por ello, en aras de su aplicabilidad, la metodología ha sido diseñada para trabajar a escalas medias, adaptadas a la escala de prevención del riesgo, no a la del proyecto de obra o informe geotécnico; la función de la metodología es alertar sobre los puntos de la infraestructura a priorizar a la hora de aplicar medidas preventivas, por tanto requiere una visión de conjunto de la casuística. La posterior actuación sobre los puntos conflictivos obligará a un análisis pormenorizado de cada caso, y a un proyecto técnico ad hoc, nivel que excede los objetivos y competencias de la metodología.

También en aras de la aplicabilidad de la metodología, se ha pretendido sacar el máximo partido a fuentes de disponibilidad general, fácil acceso y/o edición pública, y se ha procurado limitar sólo a casos imprescindibles la aportación de datos de elaboración propia o toma de decisiones abiertas por parte del técnico que la aplique. El resultado es un producto muy operativo, ya que se han automatizado la práctica totalidad de los procedimientos implicados en la metodología, y se ha dotado de agilidad a los distintos pasos de la interfaz de aplicación.

\section{Estructura de la propuesta metodológica}

A partir de los planteamientos expuestos, en la metodología se procede a predecir e identificar, en un primer paso, cuáles son los puntos potencialmente conflictivos por peligrosidad, para en un paso posterior gradar su conflictividad según la gravedad de las repercusiones de la interrupción del tráfico que el punto genera.

En razón de estos dos conceptos generales (Peligrosidad y Vulnerabilidad) se establecen dos grandes bloques metodológicos (Bloque I: Peligrosidad, y Bloque II: Vulnerabilidad), que a su vez se subdividen en módulos diferenciados temáticamente. La estructura general de la metodología queda resumida en el gráfico de la Figura 4.

Como puede observarse, el bloque de peligrosidad recoge problemas relacionados con dos grandes tipos de peligros que pueden activarse tras un episodio de lluvias torrenciales. En un primer módulo (Módulo A), se aborda la evaluación de potenciales bloqueos relacionados con movimientos gravitacionales en las laderas adyacentes a la infraestructura viaria, ya sea por alcance de materiales al firme, ya por movilización de las laderas subyacentes al mismo y descalce de la carretera. El método aborda estos problemas en tres pasos, con procedimientos diferenciados. El módulo A1 sirve para analizar la susceptibilidad a la movilización de las laderas aledañas a la infraestructura viaria, y calcular la probabilidad de movilización en ambos lados de la carretera. Se trata de un módulo complejo, que se fundamenta en un modelo predictivo causa-efecto basado en datos empíricos de movilizaciones precedentes en las áreas de estudio.

La movilización de las laderas aledañas a la infraestructura puede afectar o no a la carretera, en función, esencialmente, de la posición relativa de las laderas respecto a la calzada. Para matizar 
esta cuestión, el módulo A2 se dedica a calcular, a partir de una formulación, la probabilidad de alcance de la masa de material desplazado hasta el firme, de modo que se materialice el bloqueo. Estima, además, la magnitud del bloqueo (número viales afectados). El módulo A3 de la metodología implementa una metodología que permite abordar la probabilidad de que sea la ladera subyacente al vial la que se vea afectada por la movilización, causando con ello descalzamiento y/o subsidencia de la ladera y el firme, con el subsiguiente bloqueo del tráfico.

Figura 4. Resumen de bloques metodológicos

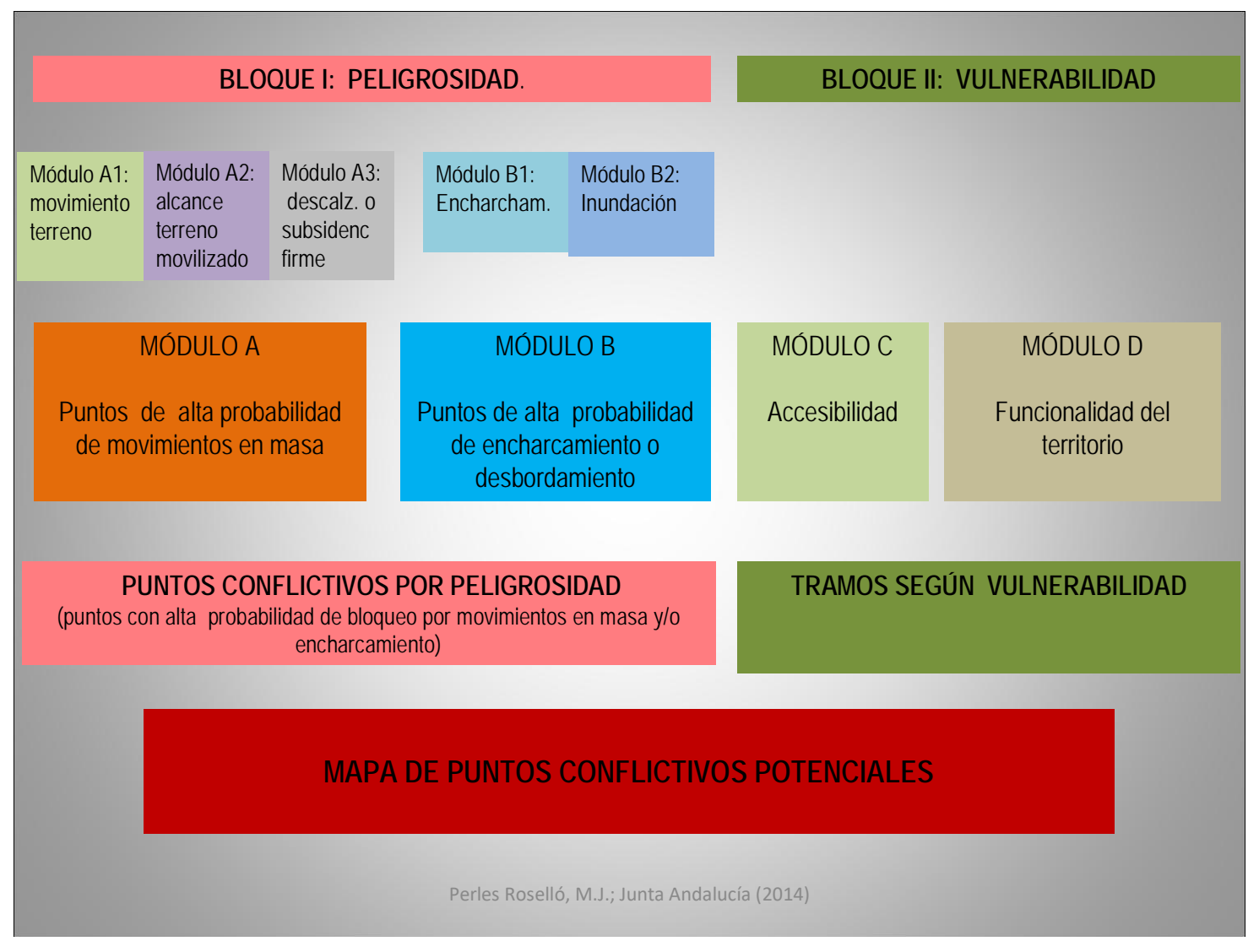

Fuente: elaboración propia

El Módulo B se dedica a la evaluación de posibles peligros relacionados con procesos hídricos. Se han distinguido dos grandes problemas: en primer lugar, la generación de balsas de agua en la carretera por encharcamiento in situ de la precipitación o problemas de drenaje del flujo hídrico acumulado (módulo B1); en segundo lugar el módulo B2 se centra en el cálculo de la posibilidad de que el agua afecte a la carretera por el desbordamiento de los cauces que la cruzan transversalmente, o discurren junto a ella.

Una vez identificada y evaluada por procedimientos específicos la distinta casuística de peligros que puede afectar una infraestructura viaria tras un episodio de lluvias torrenciales, el conjunto de puntos conflictivos por peligrosidad identificados se unifican en el mapa que aúna los puntos de alta 
probabilidad de bloqueo por movimientos en masa y los afectables por problemas de encharcamiento e inundación, síntesis del bloque de peligrosidad (Bloque I).

El Bloque II, relativo al análisis y evaluación de la vulnerabilidad, se estructura igualmente en dos módulos, uno relativo a las alteraciones de la accesibilidad y de la conectividad de la red y otro segundo relativo a las repercusiones de un posible bloqueo en la funcionalidad del territorio circundante a la red.

En el Módulo C se estiman las repercusiones del posible bloqueo o alteración del tráfico en cada punto conflictivo sobre la accesibilidad en el entorno y la conectividad de la red viaria. Se evalúa igualmente por tramos de carretera la intensidad de tráfico que puede verse alterada y la viabilidad de las rutas alternativas. En el Módulo D se desarrolla un procedimiento metodológico que permite evaluar las repercusiones del potencial bloqueo sobre la dinámica funcional del contexto territorial de la carretera. Para ello se consideran criterios como el volumen de población afectable por la interrupción del tráfico, las posibles funciones urbanas o centrales afectadas, y el volumen de equipamientos e instalaciones productivas relevantes interrumpidas. El Mapa de Tramos de carretera según su vulnerabilidad resume los resultados de accesibilidad y funcionalidad de territorio afectados obtenidos en los módulos C y D.

Por último, para obtener un valor y mapa final de puntos conflictivos, la metodología propone gradar los puntos seleccionados por su peligrosidad, y otorgarles el valor de accesibilidad y funcionalidad dictado por el tramo en el que estos puntos se sitúan. De este modo los puntos peligrosos quedan clasificados definitivamente según las repercusiones potenciales que producirán sobre la población y el normal funcionamiento del territorio.

La metodología general cuenta con procedimientos metodológicos específicos para la obtención de los resultados previstos en cada módulo. Estas metodologías sub-módulo constituyen unidades de trabajo individualizadas que difieren en sus procedimientos. Se estructuran igualmente como bloques específicos en la interfaz de aplicación. Se detallan a continuación los procedimientos metodológicos a nivel de sub-módulo, junto a los utilizados para unificar los contenidos parciales de los sub-módulos y generar, finalmente, la cartografía de puntos conflictivos.

\subsection{Bloque I (peligrosidad). Módulo A1: movimientos del terreno}

La metodología de este sub-módulo tiene como objeto evaluar la susceptibilidad a los movimientos en masa en las laderas adyacentes a la infraestructura viaria. El procedimiento de evaluación se fundamenta en un modelo determinista, en el que la susceptibilidad a la movilización del terreno se calcula en razón de la concurrencia de factores predisponentes a la inestabilidad en cada ladera. Técnicas similares han sido aplicadas por autores como Barredo et al. (2000), Razavizadeh et al., (2017), Wei Chen y Zhou (2016), o Yilmaz (2009), entre muchos otros. 
Como hipótesis de trabajo se entiende que en las laderas con signos de movilización ya constatados (eventos previos), concurren las variables que determinan en mayor medida la probabilidad de movilización del terreno, y por tanto, en sentido contrario, allí dónde concurran los factores identificados como causantes de la movilización, se podrá predecir una alta probabilidad de ocurrencia futura de movimiento del terreno. La deducción del algoritmo predictivo de la susceptibilidad a la movilización se basa en un análisis estadístico causa-efecto, un modelo de la relación de coincidencia espacial entre los factores predisponentes, que actúan como variables predictoras, y la presencia de signos indicativos de movilización en la misma zona. Se han obtenido resultados de susceptibilidad y algoritmos diferenciados para la susceptibilidad al deslizamiento y al desprendimiento (según clasificación de Varnes, 1978). De igual modo, para contrastar la validez de los resultados, se han aplicado dos procedimientos metodológicos diferentes para la deducción del modelo predictivo (un análisis basado en frecuencias y una regresión logística). Los resultados de ambos procedimientos se han comparado y se ha testeado su eficacia como predictores, para dar el modelo por válido.

Para la elaboración del modelo de indexación (Carrasco et al., 2003), se ha utilizado la ladera como unidad espacial de análisis. Se han analizado un total de 564 laderas que conforman el universo estadístico de casos para la deducción del modelo de susceptibilidad. La elaboración del modelo predictivo ha requerido llevar a cabo las siguientes fases de análisis:

- Delimitación de las laderas adyacentes a la infraestructura viaria

- Realización de un inventario de puntos de movilización del terreno históricos en las laderas de las zonas de estudio (variable dependiente).

- Identificación de los factores de susceptibilidad a incluir en el modelo (variables predictoras) y delimitación de umbrales en los intervalos intravariables.

- Aplicación de distintos procedimientos estadísticos para la indagación del modelo predictivo (análisis de frecuencias, regresión logística), y obtención del algoritmo predictivo.

- Comprobación y análisis comparado del grado de acierto de la predicción y calibración del modelo predictivo.

a) Delimitación de las laderas adyacentes a la infraestructura viaria

Las laderas se han delineado indicando su posición relativa respecto a la carretera (suprayacentes o subyacentes al vial). Para su delimitación se ha partido de un Modelo Digital de Elevaciones (MDE) de 5x5 m. de resolución (Instituto Geográfico Nacional) y ortofotos de la zona (Plan Nacional de Ortofotografía Aérea). La aplicación informática ofrece una herramienta de apoyo que permite el trazado automatizado de medias cuencas vertientes a la carretera, unidades que constituyen la base morfológica de la ladera. La ambigüedad de algunos resultados de este proceso automatizado, exige la aplicación de un procedimiento posterior de revisión y 
perfeccionamiento que se ha realizado a partir del apoyo cartografía topográfica, de pendientes, orientaciones, y sombreado de falso relieve. Se han delimitado un total de 241 laderas en las áreas de estudio.

b) Realización del inventario de puntos de movilización del terreno históricos en el área de estudio (variable dependiente)

La presencia de signos de movilización previa en las laderas de análisis constituye una evidencia empírica de su susceptibilidad al movimiento en masa, por lo que se ha considerado la variable dependiente para la deducción del modelo predictor. El inventario se ha llevado a cabo a partir de las siguientes fuentes y procedimientos:

- Trabajo de campo, y gabinete (fotointerpretación y aplicación Street View) orientado a la observación de morfologías indicativas de movilización del terreno (erosión o depósito) en las laderas.

- Recopilación de informes de incidencias relacionados con la movilización de laderas adyacentes a la infraestructura, procedentes de la Dirección General de Carreteras.

- Presencia de obras artificiales de control de taludes indicativas de movimientos precedentes.

c) Identificación de los factores de susceptibilidad a incluir en el modelo (variables predictoras) y delimitación de umbrales en los intervalos intravariables

La identificación de factores predisponentes a la movilización se ha basado en criterios bibliográficos, y en la observación directa de factores desencadenantes artificiales que son propios del entorno transformado de la infraestructura. Entre las variables causantes se han descartado las relacionadas con la precipitación o la sismicidad como activadores del movimiento, ya que, como observan Bornaetxea et al. (2016), no resultan discriminantes espacialmente a la escala de detalle que se ha utilizado, en la que presentan un comportamiento constante, y no variable.

Los variables identificadas como predictoras de la susceptibilidad son las siguientes:

- Estabilidad en vertientes de la litología

- Homogeneidad interna de la serie litológica

- Presencia y tipo de contactos litológicos

- Presencia y tipo de discontinuidad tectónica

- Orientación del plano potencial de movilización

- Pendiente de la ladera

- Disposición de la calzada o ladera aledañas sobre formas geomorfológicas activas

- Presencia de agua en la ladera

- Presencia y tipo de vegetación 
- Signos de remoción natural o artificial en la ladera

- Presencia de talud artificial

Algunas variables seleccionadas por su demostrada incidencia en la movilización (agua en la ladera, vegetación, geomorfología activa u orientación de la estructura favorable a la caída) han sido apartadas del modelo predictivo por motivos diversos de representatividad estadística (escasa representación y/o variabilidad en el área de estudio, lo que proporciona datos de correlación con la variable dependiente muy bajos). En estos casos, las variables han sido incluidas en el modelo de evaluación final como condición directa de inestabilidad, que eleva directamente el valor de susceptibilidad al intervalo mayor.

d) Elaboración del modelo predictivo (análisis de frecuencias, regresión logística)

Se han elaborado dos estrategias metodológicas para deducir el algoritmo predictor de la probabilidad de movilización.

En la primera de ellas se ha aplicado un procedimiento que pretende identificar cuál es el patrón de factores causantes presente en las laderas que ya han demostrado que son inestables. El objetivo es conocer qué variables predictoras inciden en mayor o menor medida en la génesis de la caída, para así ordenar su nivel de participación en la probabilidad de caída mediante la asignación de pesos proporcionales a su rol. Se ha entendido que las variables que inciden de manera preferente en la movilización serán aquellas que, observadas en el grupo de las laderas manifiestamente inestables (grupo de laderas movilizadas), aporten con más frecuencia valores máximos de inestabilidad. El valor de repetición del intervalo de máxima inestabilidad para cada variable ha sido estandarizado y utilizado como coeficiente de ponderación de la misma, esto es, como indicador de su contribución a la inestabilidad. A partir de estos datos, el algoritmo final predictivo obtenido se configura como una sumatoria lineal ponderada, para los casos de desprendimientos, en los siguientes términos:

$$
\begin{aligned}
& \mathrm{SDP}=(\text { LIT } \times 0,27)+(\text { PEND } \times 0,22)+(\text { HET } \times 0,17)+(\text { TAL } \times 0,14)+ \\
& (\text { CONT } \times 0,11)+(\text { DISC } \times 0,09)
\end{aligned}
$$

Siendo SDP = Susceptibilidad a desprendimientos; LIT = Litología; PEND = Pendientes; HET = Heterogeneidad interna de la serie litológica; TAL = Taludes en ladera; CONT = Presencia de contactos litológicos; DISC = Presencia de discontinuidad tectónica.

Respecto a los deslizamientos, el algoritmo generado tiene la siguiente estructura:

$$
\begin{aligned}
& \text { SDZ }=(\text { LIT } \times 0,32)+(\text { PEND } \times 0,25)+(\text { HET } \times 0,20)+(\text { TAL } \times 0,10)+ \\
& (\text { CONT } \times 0,08)+(\text { DISC } \times 0,05) .
\end{aligned}
$$


Siendo SDZ = Susceptibilidad a desprendimientos; LIT = Litología; PEND = Pendientes; HET = Heterogeneidad interna de la serie litológica; TAL = Taludes en ladera; CONT = Presencia de contactos litológicos; DISC = Presencia de discontinuidad tectónica.

Los algoritmos obtenidos han sido utilizados finalmente para generar mapas predictivos de susceptibilidad al desprendimiento.

La segunda estrategia utilizada para deducir el algoritmo predictivo ha sido la aplicación de una regresión logística. La regresión logística binomial permite deducir una ecuación que establece la relación matemática subyacente entre una variable dicotómica dependiente, y un conjunto de variables predictoras independientes (Hosmer \& Lemeshow, 2004).

Se ha utilizado como variable dependiente de la regresión logística el inventario de laderas con signos de movilización, que tiene una estructura binaria (laderas movilizadas o no movilizadas). Los variables predictoras, en este caso, son las indicadoras de susceptibilidad. Una vez realizado el análisis, obtenidos los coeficientes y analizada su significación estadística, la ecuación obtenida permite realizar mapas de probabilidad de caída con carácter predictivo.

e) Análisis comparado del grado de acierto de la predicción y validación del modelo predictivo Una vez obtenidos los modelos predictivos mediante los distintos procedimientos reseñados, se ha evaluado la correspondencia entre los valores reales de laderas caídas y los predichos a través de distintas estrategias de análisis. Además de valorar el nivel de coincidencia a través de porcentajes de acierto en los distintos intervalos de gravedad, se ha elaborado una matriz de confusión, que enfrenta los valores reales y los predichos. Se ha dibujado igualmente una curva ROC (Reciever Characteristics Curve), que representa el porcentaje de positivos correctos que un modelo predice según el corte de probabilidad es bajado desde 1 hasta 0 . Se ha comprobado que la capacidad predictiva de los modelos obtenidos mediante distintos métodos es similar, con valores de AUC en torno a 0,8 , y se confirma una distribución de pesos similar entre las distintas variables predictoras para los algoritmos comparados. Los valores de acierto en la estimación de las laderas caídas pueden calificarse como muy positivos (Bormaetxea et al., 2016) y permiten validar la capacidad predictiva del modelo.

La interfaz informática diseñada permite la aplicación del modelo y la elaboración automatizada del conjunto de procedimientos necesarios para aplicar el Módulo A1, esto es, para calcular la susceptibilidad de movilización en cada ladera, y con ello su peligrosidad (ver Figura 5). 
Figura 5. Peligro de deslizamientos y desprendimientos en laderas adyacentes a la carretera

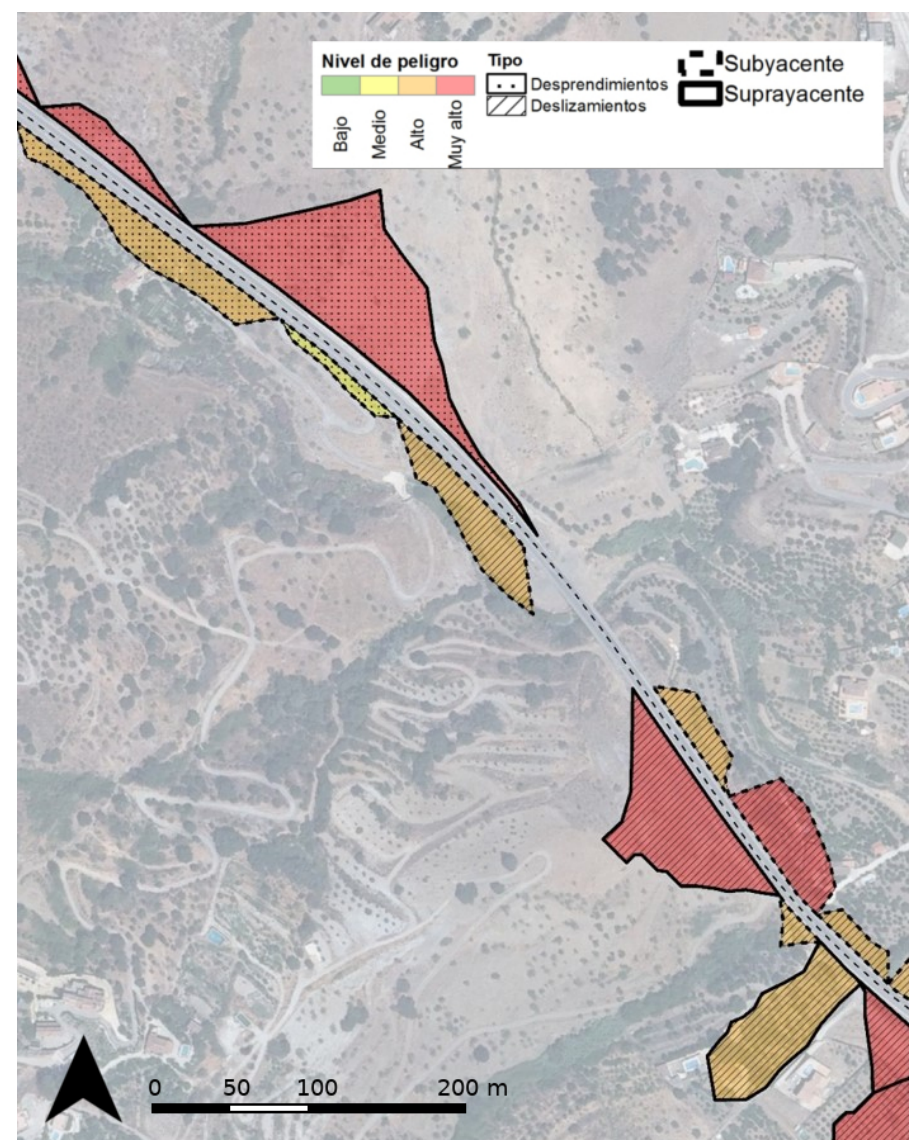

Fuente: elaboración propia

\subsection{Bloque I (peligrosidad). Módulo A2: alcance del terreno movilizado}

En el módulo A2 se desarrolla un procedimiento metodológico destinado a evaluar la posibilidad de que el material movilizado por movimientos en masa en las laderas suprayacentes a la carretera llegue a alcanzar la carretera en su desplazamiento. Para ello se siguen los siguientes pasos:

- Cálculo de la distancia potencial de desplazamiento de la masa movilizable en cada ladera.

- Cálculo de distancia entre la base de la ladera y la calzada en cada punto.

- Cálculo de la posibilidad de afectación de la carretera por la masa desplazada en uno o dos viales, a partir de la relación de los parámetros anteriores.

Para calcular la distancia potencial que recorrerían los materiales movilizados se ha utilizado la fórmula propuesta por Nilsen (2008), que calcula la distancia de alcance a partir de variables de fácil obtención. Se han desechado fórmulas más completas pero más exigentes en la precisión de los datos de entrada tales como las propuestas por Ikeya (1989), Rickenmann (1999) o Zhang et al. (2013). La fórmula de Nilsen (2008) deriva de una correlación observada entre el ángulo de talud 
$(\Psi)$, y el ángulo formado por la línea trazada entre la cima de la ladera y el punto que alcanza el material desprendido $(\beta)(R 2=0.80)$

$$
\beta=3.926+0.768 \Psi
$$

En nuestro modelo asimilamos que el ángulo $\Psi$ es el formado por la ladera con un hipotético plano horizontal al pie de la misma. Este ángulo se obtiene de forma automatizada a partir del mapa de pendientes, y, mediante relaciones trigonométricas, se calcula la proyección horizontal de la distancia que recorrerían los materiales movilizados. Una vez obtenida la distancia potencial de alcance, éste dato se ha comparado, en cada ladera, con la distancia que existen desde el borde de la ladera hasta la línea exterior de la carretera (borde de la calzada) y el eje central de la misma. En función de la relación que exista entre ellas y la posibilidad de bloquear una o dos calzadas/carriles, se asignan las diferentes clases de probabilidad de alcance. En la aplicación informática, se ha incluido un módulo que calcula, de forma automática, todos los valores necesarios para el cálculo, a partir de los datos procedentes del Modelo Digital de Elevaciones.

\subsection{Bloque I (peligrosidad). Módulo A3: descalce y/o subsidencia del firme}

El objetivo de este módulo es seleccionar todas aquellas laderas que reúnan condiciones favorables al descalce del firme que sustenta la carretera. La principal condición causante de este fenómeno es la inestabilidad de la ladera en la que reposa la calzada. Tras la observación en campo de la casuística, se han seleccionado también como causas de movilización y descalce la presencia de zonas de encharcamiento y balsas en la base de la ladera sustentante (ver figura 6), con la consiguiente humectación, así como la identificación de signos de erosión stricto sensu (remoción y vaciado de material) en la misma, tanto de origen natural como artificial. El algoritmo de selección en el que se basa la identificación de la probabilidad de descalce en el Módulo A3, se basa en criterios de selección booleana a partir de las variables citadas. 
Figura 6. Peligro de descalce por encharcamiento basal y/o remoción

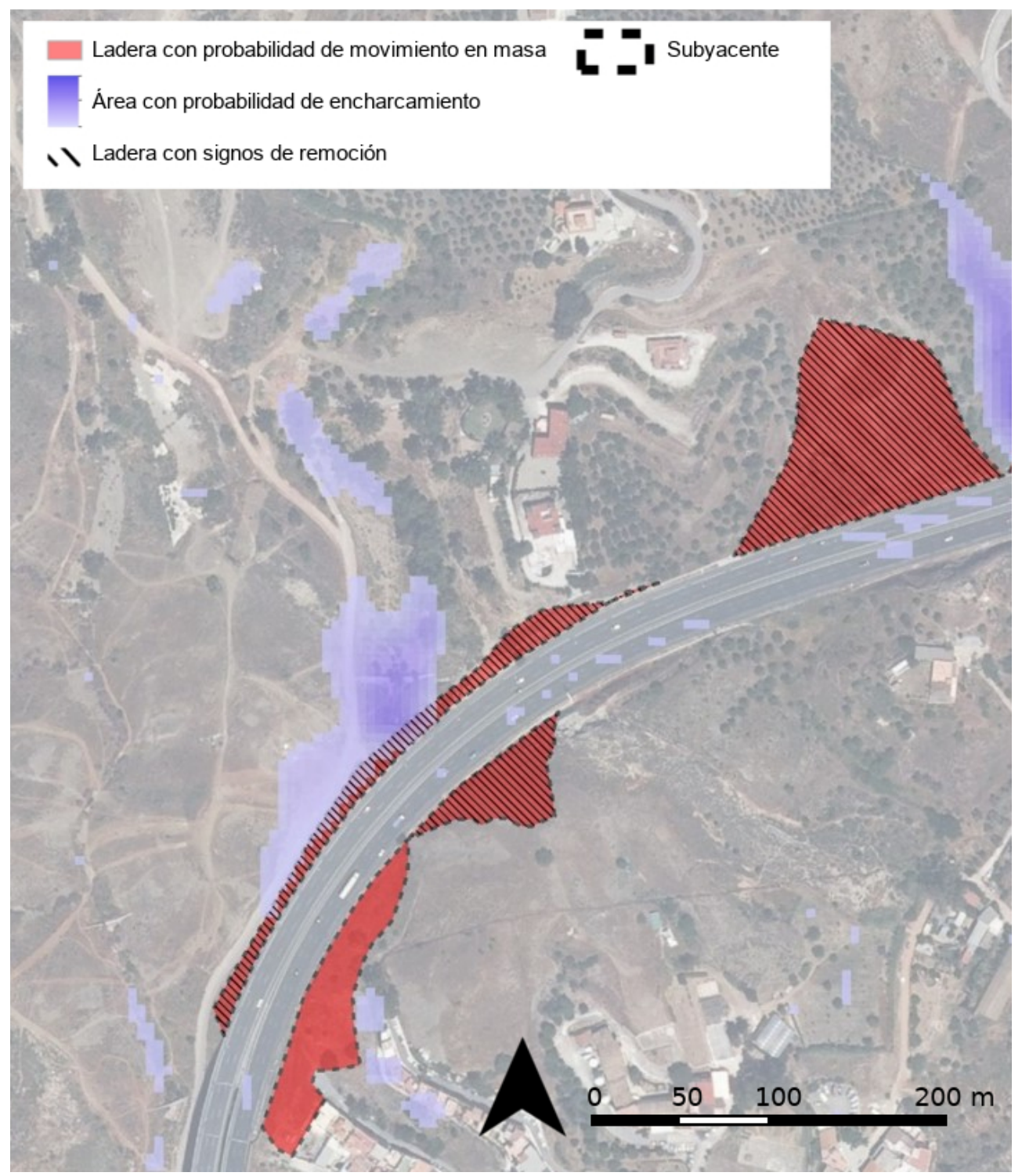

Fuente: elaboración propia

\subsection{Bloque I (peligrosidad): resultados conjuntos del Módulo A}

Para evaluar la probabilidad de afectación de la calzada por movimientos del terreno (alcance desde laderas suprayacentes y descalzamiento desde laderas subyacentes), la metodología resume las condiciones más desfavorables de los procedimientos aplicados en los módulos A1, A2 y A3. Para su representación cartográfica, aplica un procedimiento de transferencia espacial desde las laderas causantes de la afección en la carretera, hasta la carretera misma, para así definir sectores de la carretera con mayor probabilidad de afección (ver Figura 7). 
Figura 7. Mapa final resultante Módulo A

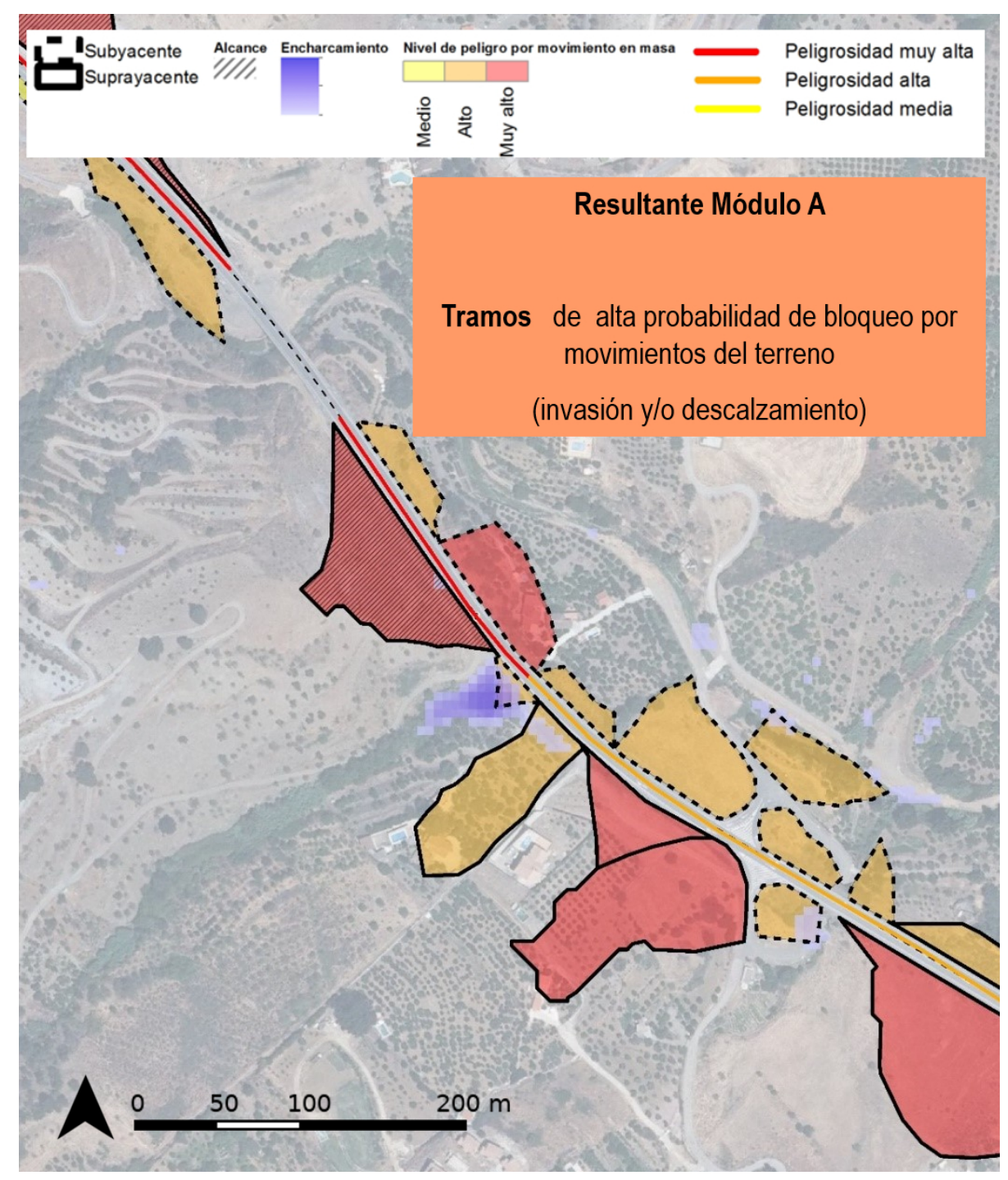

Fuente: elaboración propia

\subsection{Bloque I (peligrosidad). Módulo B1: puntos de alta probabilidad de encharcamiento o desbordamiento}

El módulo B1 aporta una metodología que evalúa la probabilidad de encharcamiento por generación de balsas de agua de las infraestructuras viarias. Para que se forme una balsa tiene que darse una circunstancia de acumulación de agua y/o déficit de drenaje, que en ocasiones se deriva de la propia intensidad de la precipitación (acumulación in situ), o del atasco del sistema de drenaje artificial de la infraestructura por acumulación de aportes sólidos en los culverts. Para la balsa se configure, es necesario igualmente que exista en la calzada una morfología subsidente que retenga el agua. A partir de estos condicionantes, la metodología del módulo B1 está orientada a obtener información de las variables que se exponen a continuación:

- condiciones de subsidencia topográfica en el perfil longitudinal de la carretera (micro-cuencas endorréicas); 
- disponibilidad y circulación del flujo de escorrentía hacia el punto subsidente; y

- probabilidad de atasco en el sistema de drenaje por incorporación de aportes sólidos al flujo de escorrentía

En primer lugar, la metodología prevé la detección de zonas endorreicas (o "sinks", en la terminología de los SIGs) en la carretera. Para ello, se requiere un MDE con suficiente nivel de detalle, y en el que estén adecuadamente representadas las transformaciones introducidas por el trazado de la carretera. Como referencia, puede usarse el Modelo Digital del Terreno de $5 \mathrm{~m}$. del Instituto Geográfico Nacional. Una vez obtenidas las zonas endorreicas para toda el área de estudio, se seleccionan aquellas situadas en el interior de la carretera y que coinciden con puntos cóncavos del perfil longitudinal de la misma. Finalizada esta operación, ya se conoce cuáles son las posibles áreas de la carretera susceptibles de inundarse.

Para que tales zonas se inunden realmente es preciso que por ellas circule escorrentía que pueda llegar a ellas. Para conocer este dato, el siguiente paso metodológico consiste en determinar el caudal procedente de las laderas suprayacentes a la carretera, así como de las cuencas que vierten al sistema de drenaje lateral de la misma. La escorrentía por unidad de superficie generada por cada ladera o cuenca es la diferencia entre el valor de precipitación y la media de ese coeficiente de escorrentía calculada para el área de cada unidad espacial. ${ }^{3}$

Una vez que se conoce la escorrentía producida por cada cuenca o ladera, su valor se lleva al eje de la carretera, y desde este punto se procede a simular la circulación del flujo hídrico por la carretera. Considerando la situación más desfavorable, se parte de la hipótesis de que todos los sistemas de drenaje se han bloqueado, y por tanto la escorrentía circula libremente por la carretera. ${ }^{4}$ La aplicación determina las direcciones de flujo en función de la disposición de los puntos cóncavos y convexos, así como la altura media de cada segmento, y va acumulando el caudal procedente de cada unidad adyacente (cuencas y laderas). Los puntos cóncavos suponen los nodos de captación de ese sistema, en los que terminan las acumulaciones de flujo, mientras que los convexos las reinician. Este fenómeno se representa, en la cartografía automatizada resultante, mediante una línea cuyo grosor es proporcional al caudal que circula por él. Conocido el caudal máximo teórico que circularía por cada tramo, se descartan aquellas áreas endorreicas que corresponden a segmentos de caudal nulo, y se seleccionan las restantes como posibles puntos de encharcamiento. El caudal acumulado en cada segmento de la carretera podría utilizarse

3 El cálculo de la escorrentía de cuencas y laderas suprayacentes a la calzada se ha obtenido a partir del método del número de curvas (Soil Conservation Service, USA). Su aplicación en España está regulada por la Instrucción 5.2-IC de Drenaje Superficial, del Ministerio de Fomento (2017).

4 Se parte de la situación de bloqueo de drenaje más desfavorable para fijar como constante esta variable, y así poder identificar, en términos comparativos, cuáles son los tramos de la carretera con mayor potencial de recibir escorrentía y carga sólida desde sus laderas y cuencas vertientes. No se pretende averiguar a escala de detalle si los sistemas de drenaje artificial de la carretera están bien dimensionados para el caudal y carga a evacuar. 
también como un indicador de la posibilidad de aquaplaning, en caso de que el firme no tuviese suficiente capacidad de drenaje.

El módulo de encharcamiento de la aplicación también calcula, para cada cuenca y ladera suprayacente, el aporte de sedimentos y de residuos vegetales que llegarán a los sistemas de drenaje transversal y longitudinal de la carretera, y con ello permite aproximarse a la posibilidad de atascos en los sistemas de drenaje artificiales de la infraestructura.

Para obtener este valor se parte de datos de erosión ( $\mathrm{tm} / \mathrm{ha}$ /año de material movilizado), residuos vegetales y biomasa producidos como media por cada cultivo y especie vegetal, y posibles arrastres de gran tamaño (mobiliario urbano), que en el área de estudio se han obtenido de la REDIAM (Red de Información Ambiental del Andalucía) y del SIOSE (Sistema de Información sobre Ocupación del Suelo de España).

A partir de estos datos se calcula la producción media de escorrentía, sedimentos y residuos vegetales por unidad de superficie, y una relación de la proporción entre carga sólida y el volumen líquido en el flujo de escorrentía. Estos datos suministran permiten detectar qué laderas o cuencas vertientes son responsables en mayor medida de posibles atascos en los drenajes artificiales de la infraestructura.

Todo el proceso expuesto en el módulo ha sido implementado en la interfaz de aplicación, que facilita la identificación automatizada de los puntos en los que la escorrentía se embalsa, calcula la acumulación de flujo hídrico en cada tramo de la calzada, y posibilita igualmente el cálculo automatizado del porcentaje de carga sólida presente en el caudal de escorrentía generado por cada ladera o cuenca vertiente. Al calcular la altura de la lámina de agua sobre la rasante, permite establecer umbrales de peligrosidad por profundidad del calado.

\subsection{Bloque I (Peligrosidad). Módulo B2: inundación del firme por desbordamiento de cauces aledaños}

El módulo B2 aporta una metodología que estima la probabilidad de que el caudal de inundación de los cauces aledaños a la calzada, llegue a alcanzar e inundar a la misma, afectando su funcionalidad.

La aplicación calcula de forma automatizada la probabilidad de que el caudal de crecida llegue a inundar la calzada a partir de datos de caudales estimados de forma externa a la aplicación que permiten, mediante un modelo hidrológico-hidráulico unidimensional, deducir la altura de la lámina de agua prevista en un episodio torrencial tipo. Estos datos se ponen en relación con el desnivel existente entre el punto de cruce de la carretera y la altura del cauce en cuestión, para así evaluar la probabilidad de alcance del agua a la calzada. 
En el cálculo de caudales, se ha procurado ajustar los cálculos de caudal y de calado de los cauces a la realidad del entorno mediterráneo, en el que se producen crecidas, compuestas en gran medida por arrastres sólidos incorporados al caudal. Los aportes sólidos alteran de forma radical el volumen del caudal, y pueden generar atascos y retenciones que incrementan de forma drástica la probabilidad de desbordamiento y alcance de la carretera. Por este motivo, en la metodología se ha considerado la incidencia de las características de la cuenca en lo que respecta a acarreos potenciales procedentes de la erosión hídrica, de movimientos en masa, de restos vegetales y de otras posibles cargas artificiales que puedan incorporarse al caudal (automóviles, mobiliario urbano, etc.). El estudio hidrológico-hidráulico ha incorporado el volumen de residuos sólidos que llega a arrastrar el flujo. Una vez evaluados los sedimentos sólidos se transforman a un valor de volumen $\left(\mathrm{m}^{3}\right)$, se calcula su relación de proporción con respecto al total de caudal escurrido durante la lluvia $\left(\mathrm{m}^{3}\right)$, y se incrementa el caudal con el que se hace el estudio hidráulico en esa misma proporción (\%). A partir de este dato de volumen de caudal modificado, se calcula el calado de la crecida, y la altura a la que llegará la lámina de inundación. Este valor se pone en relación con la altura de la carretera en dos puntos: la base del talud sustentante y en la propia calzada. A partir de estos datos, se identifican los puntos de la infraestructura con mayor probabilidad de ser inundados por desbordamiento de cauces transversales o paralelos ${ }^{5}$

Las variables que intervienen en este módulo, son, por tanto, las siguientes:

- Cálculo del caudal de inundación tipo para los cauces aledaños y/o transversales a la infraestructura viaria

- Incorporación de los posibles aportes sólidos procedentes de la cuenca al volumen de caudal líquido

- Cálculo de la altura máxima del caudal de crecida con incorporación de los acarreos sólidos

- Relación de la altura de la lámina de agua con la del punto de intersección entre el cauce y la carretera.

\subsection{Bloque I (Peligrosidad). Módulo B (síntesis): puntos de alta probabilidad de encharcamiento o desbordamiento}

Al igual que con el modulo A, la metodología resume en esta fase las condiciones probables más desfavorables de las fases metodológicas B1 y B2, identificando aquellos sectores de la infraestructura viaria con alta probabilidad de presentar problemas relacionados con el agua (encharcamiento, aquaplaning o desbordamiento).

5 Los valores de nivel de erosión de material en las cuencas vertientes, producción de sedimentos y carga sólida han sido calculados para una escala media, a partir de la RUSLE (Revised Universal Soil Loss Equation). Se han utilizado métodos adaptados a esta escala, basados en fuentes de información editadas (no trabajo de campo), y, por ello, replicables en posteriores aplicaciones del método a través de la interfaz de aplicación automatizada. Es el caso de la RUSLE. 


\subsection{Bloque II (Vulnerabilidad). Módulo C: alteraciones de la accesibilidad de los distintos tramos de la red viaria}

El Bloque II de la metodología general se dedica al análisis de la vulnerabilidad de la carretera y el territorio afectado por los posibles bloqueos del tráfico. Contiene dos módulos, referentes a alteraciones de la accesibilidad y de la conectividad de la red (módulo C), y a las repercusiones del potencial bloqueo en los servicios territoriales del territorio circundante a la red (módulo D).

El primero de ellos (módulo C) recoge un procedimiento metodológico que evalúa la accesibilidad de la infraestructura viaria en los distintos sectores de la red. El objetivo último del módulo es calibrar la relevancia que tendría el bloqueo hipotético de un eje en función del lugar donde se produzca el bloqueo. El análisis de la accesibilidad de la red en cada tramo permite obtener una idea de las repercusiones que tendrá el bloqueo según provoque, en atención a su posición, alteraciones mayores o menores de la accesibilidad en la zona.

Para acometer este bloque de la metodología, una vez delimitada la red, se ha definido como unidad de análisis el tramo de carretera. El tramo se ha definido como el segmento que, tomando como referencia un nodo, abarca desde la mitad de uno de los arcos que parten del nodo en un sentido hasta la mitad del arco que parte del nodo en sentido contrario. Por ello, los objetos que conectan con la red a través de un nodo son asignados a un único tramo, lo que facilita el análisis. Al mismo tiempo, cada tramo alberga en su interior un único nodo.

Se ha entendido que las consecuencias de un potencial bloqueo del tráfico serán más o menos conflictivas según su incidencia en la conectividad del tramo de carretera afectado, en la intensidad del tráfico interrumpido, y según sea la funcionalidad efectiva de las rutas alternativas al tramo bloqueado, medida a través de la velocidad de circulación que éstas permitan. Estos factores han sido incluidos en la metodología e interfaz de aplicación como se recoge a continuación:

- Conectividad del tramo de carretera afectado. La importancia de cada tramo en la red se ha medido a través del número de arcos de la red conectados con el tramo en cuestión, en ambos sentidos del tráfico (ver figura 8)

- Intensidad del tráfico bloqueado. La intensidad del tráfico potencialmente susceptible de bloqueo se ha analizado utilizando como indicador la Intensidad Media Diaria (IMD), tanto la diaria como la calculada en hora punta.

- Viabilidad de rutas alternativas. Para analizar la viabilidad de las rutas alternativas de cada tramo se han utilizado tres variables: el índice de rodeo, la intensidad de tráfico del recorrido alternativo (intensidad media diaria) y la jerarquía de las vías alternativas. 
Figura 8. Vulnerabilidad de tramos de carretera según su conectividad

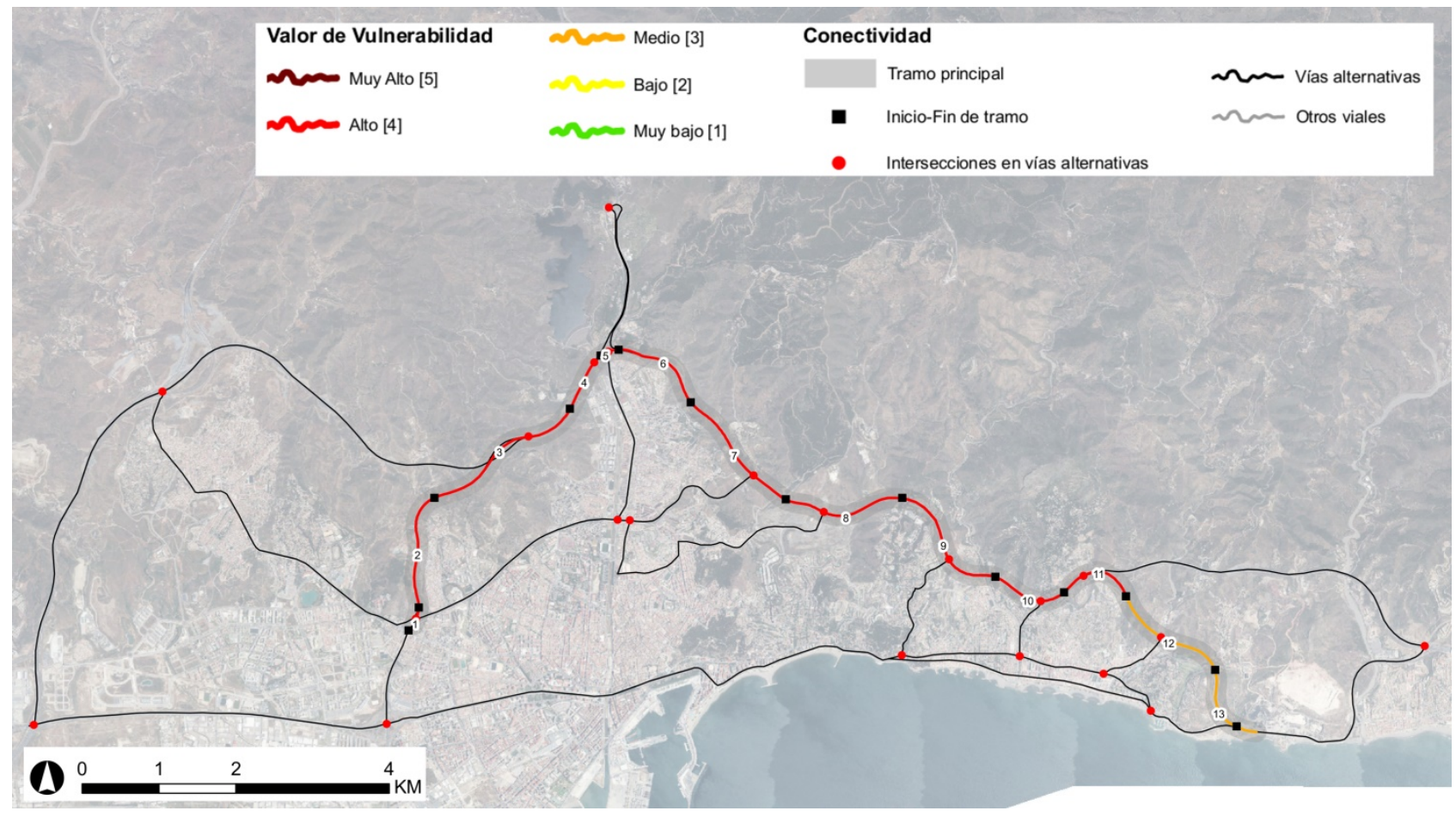

Fuente: elaboración propia

A partir de estas variables, se han obtenido valores conjuntos de vulnerabilidad según la accesibilidad del tramo mediante una sumatoria lineal ponderada. Los coeficientes de ponderación para cada variable han sido asignados a partir de criterios bibliográficos y de subjetividad compartida. La asignación de intervalos de gravedad en cada variable, los pesos y el procedimiento de cálculo conjunto han sido incorporado a la aplicación informática.

\subsection{Bloque II (Vulnerabilidad). Módulo D: repercusiones del bloqueo en la funcionalidad del territorio atendido por la red viaria}

El módulo D desarrolla una estrategia metodológica que permite evaluar la participación de los distintos tramos de la red viaria en la funcionalidad del territorio circundante al que éstos dan acceso. A partir de este dato, se pretende evaluar las repercusiones territoriales que podría acarrear el bloqueo del tráfico en cada uno de los tramos, esto es, la magnitud y trascendencia, cualitativa y cuantitativa, de las funciones territoriales alteradas o interrumpidas. Se entiende que un tramo de carretera será más vulnerable cuando su bloqueo repercuta en servicios y funciones del territorio más perentorias y/o estratégicas, y afecten a una mayor cantidad de población. Según este planteamiento, la vulnerabilidad del territorio se ha valorado en razón del volumen de población afectable, la trascendencia de las funciones urbanas potencialmente alterada, y el volumen de equipamientos e instalaciones productivas relevantes interrumpidas.

Se exponen a continuación, de forma más precisa, las variables consideradas en el módulo D: 


\section{a) Población residente total afectada por el bloqueo del tráfico}

Se han clasificado los núcleos de población afectables en función de la relación que tiene sus accesos con respecto a la red estudiada. De este modo se intenta delimitar y clasificar el nivel de dependencia que tiene la población del núcleo respecto al tramo o tramos de carretera que da acceso al mismo. Se han clasificado los núcleos según su acceso a la red viaria en estudio sea preferente o secundario, y en razón del carácter interno o externo del núcleo respecto a la red. Se han considerado núcleos externos a la red los situados fuera de la red, pero que tienen una vinculación funcional con la misma, siendo el eje principal u otros elementos de la red accesos importantes para estas poblaciones. La población de estos núcleos está afectada por el posible bloqueo del tráfico en menor medida, por estar vinculada a otras redes, pero su movilidad está relacionada de alguna manera con la red estudiada.

Los núcleos se organizan, en definitiva, en tres categorías:

- Accesos importantes internos a la red

- Accesos secundarios internos a la red

- Accesos importantes externos a la red

En función de estas categorías, se pondera la población de los núcleos por un determinado coeficiente, en función de la mayor dependencia que tiene cada núcleo de población respecto al uso de la red. En un paso final, se asignan los resultados de población afectable obtenidos en cada núcleo al tramo del eje principal al que esté vinculado el núcleo, sumándose los resultados obtenidos en cada tramo.

b) Asentamientos de población según sus funciones territoriales

El reclamo de movilidad hacia los diferentes asentamientos humanos está estrechamente ligado a su jerarquía en el sistema de ciudades. Los núcleos urbanos de mayor nivel jerárquico, cuentan con un mayor número de equipamientos y prestan mayores servicios, tanto a su población como a los asentamientos que se encuentren en su área de influencia. Por este motivo, los bloqueos de tráfico en tramos que den acceso a núcleos de población de mayor importancia funcional producen impactos de mayor trascendencia, y, en consecuencia, otorgan al tramo una mayor vulnerabilidad. En el trabajo, la jerarquización y asignación de coeficientes de ponderación de los asentamientos y tramos de carretera correspondientes se ha establecido siguiendo la "Jerarquía del Sistemas de Ciudades de Andalucía", recogida en el POTA (Plan de Ordenación del Territorio de Andalucía). La puntuación correspondiente a la funcionalidad urbana, a su vez, es matizada según el núcleo tenga un acceso preferente o secundario a la red.

c) Afección a equipamientos e instalaciones productivas interurbanas 
Los equipamientos e instalaciones productivas situados fuera de los núcleos generan un flujo de desplazamientos por sí mismos, tanto de trabajadores como de usuarios o consumidores muy sensible al potencial corte de tráfico. Se han distinguido dos niveles de vulnerabilidad en razón de la importancia de las funciones urbanas potencialmente alterable, según se trate de equipamientos básicos y actividades económicas fundamentales, o de carácter secundario. En el grupo de máxima vulnerabilidad se han incluido equipamientos tales como grandes centro de transportes, emergencias, sanitarios, grandes infraestructuras de saneamiento y abastecimiento de agua y energía y centros penitenciarios; se han incluido también como instalaciones productivas de mayor vulnerabilidad frente al corte de tráfico las Áreas Logísticas, los espacios productivos en funcionamiento ocupación mayor al $50 \%$, los grandes centros comerciales y los mercado de abastos externos al núcleo de población. La importancia de la vulnerabilidad en cada caso ha sido ponderada igualmente por la posición de la instalación dentro o fuera de la red. Los datos se han obtenidos de la cartografía del Datos Espaciales de Referencia de Andalucía (DERA), del Instituto de Estadística y Cartografía de Andalucía, donde están cartografiados los equipamientos e instalaciones productivas analizadas. Para localizar los hoteles, que no se encuentran en la base de datos del DERA, se ha procedido a hacer una búsqueda a través de Google Earth.

En un último paso, se calcula un valor unitario del Módulo D, valor que expresa la vulnerabilidad de cada tramo de la carretera en razón de las consecuencias que un bloqueo del tráfico tendría sobre las funciones urbanas del entorno. Se propone para ello una suma ponderada de las variables del módulo en atención a los siguientes coeficientes:

- Población residente total afectada (35\%)

- Funciones territoriales de los núcleos (25\%)

- Equipamientos y actividades económicas interurbanas (40 \%)

\subsection{Metodología final de unificación (peligrosidad + vulnerabilidad): mapa de puntos conflictivos potenciales}

El Mapa de puntos conflictivos constituye el mapa final resultante del proyecto y objetivo fundamental del mismo. En él se resumen todos los puntos conflictivos y quedan integrados los factores de peligrosidad y vulnerabilidad estudiados.

El mapa se ha construido a partir de la selección de los puntos de máxima peligrosidad, que, según el tramo de vulnerabilidad en el que se sitúen, quedan clasificados en tres niveles finales de riesgo, valores que indican finalmente el grado de conflictividad del punto en cuestión. 
Figura 9. Mapa de puntos conflictivos potenciales

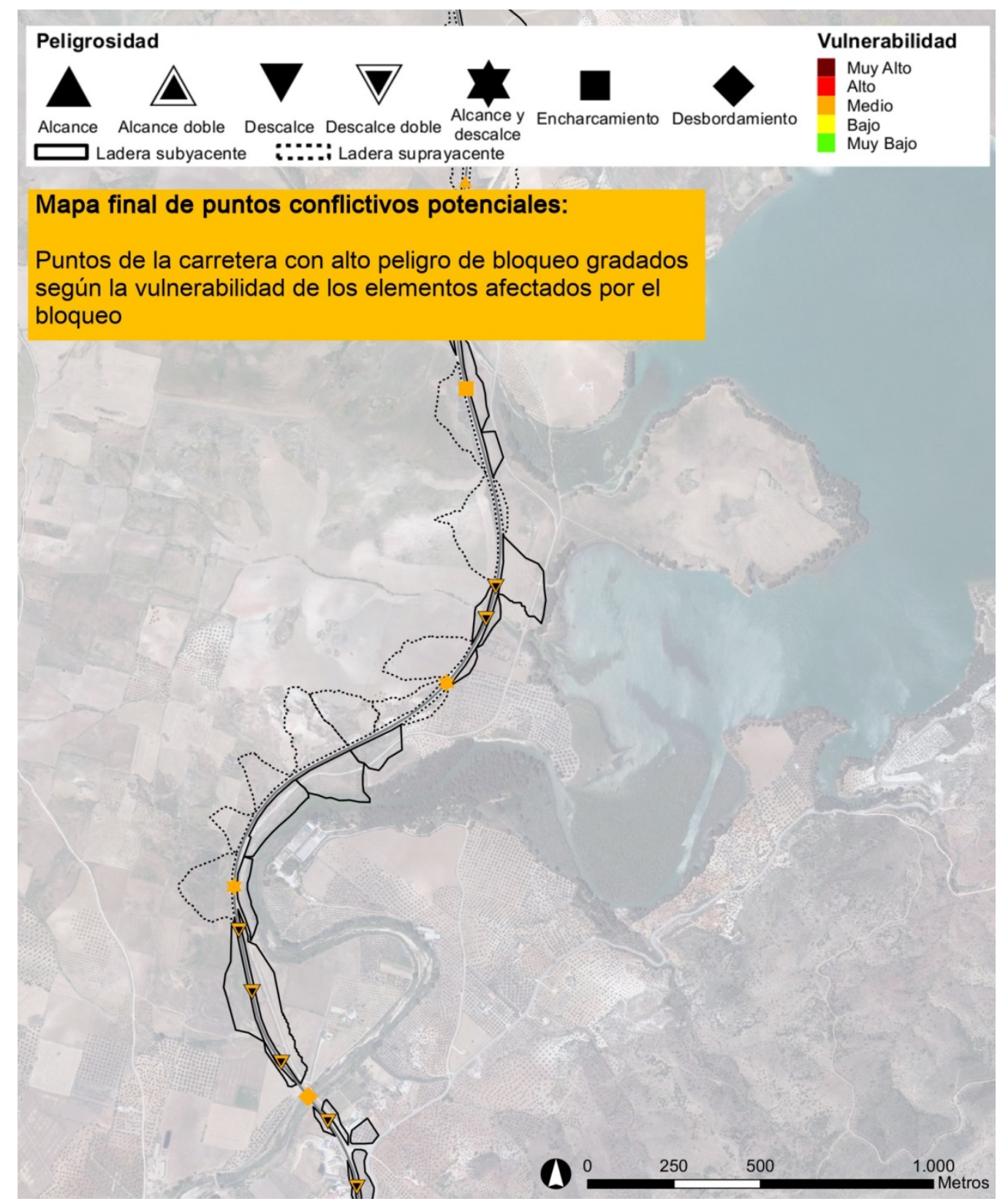

Fuente: elaboración propia

La expresión cartográfica (ver Figura 9) se ha resuelto utilizando símbolos para identificar el tipo de problema de peligrosidad que produce el bloqueo (alcance de la carretera por movimiento en masa, descalce de la misma por la misma causa, encharcamiento o desbordamiento). Se ha considerado igualmente la probabilidad de que en un punto se reunan problemas de peligrosidad en los dos sentido de la carretera, o sólo en uno. El simbolo que representa el tipo de problema de peligrosidad potencial se reproduce en distinto color, según el grado de vulnerabilidad del tramo de carretera en el que el punto se ubica sea mayor o menor. 


\section{Diseño de la interfaz de la aplicación}

Como producto final, se ha diseñado una interfaz informática para la aplicación del modelo predictivo a otras infraestructuras viarias. La interfaz ha sido diseñada en lenguaje de programación Grass, compatible con software libre de Sistemas de Información Geográfica (SIG) que posibilita la elaboración automatizada del conjunto de procedimientos espaciales y cálculos numéricos necesarios para estimar y cartografiar la probabilidad de movilización del terreno en laderas adyacentes a infraestructuras viarias. En aras de su aplicabilidad, la metodología ha sido diseñada (ver Figura 10) para sacar el máximo partido a fuentes de disponibilidad general, fácil acceso y/o edición pública, y se ha procurado limitar sólo a casos imprescindibles la aportación de datos de elaboración propia o toma de decisiones abiertas por parte del técnico que la aplique. La automatización de la práctica totalidad de los procesos, y la agilidad y claridad en el procedimiento que permite la interfaz de aplicación contribuye de forma directa a la obtención de un producto resultante eficaz para la aplicación de la metodología.

\section{Conclusiones}

Como se ha reflexionado en el artículo, el entorno de las infraestructuras viarias constituye un espacio de riesgo singularizado por concentrar en el espacio procesos de peligrosidad de distinta etiología y funcionamiento, por poseer una morfología compleja, en tanto que mixta y transicional entre lo natural y lo artificial, y por ofrecer un elemento en riesgo, el tráfico viario, de carácter estratégico, especialmente en los momentos de crisis (retornos, refugio, operaciones de socorro, asistencia o rescate).

En este contexto, se ha conseguido con éxito caracterizar el funcionamiento particular de los procesos de peligrosidad al adaptarse al entorno antropizado de la infraestructura y deducir un modelo predictivo de la peligrosidad de origen múltiple adaptado a esta casuística. De igual forma, se ha resuelto de forma eficaz la estrategia metodológica para evaluar la vulnerabilidad de la infraestructura frente al bloqueo del tráfico, superando el nivel de la simple estimación de costes de reparación, hacia la evaluación de impactos potenciales de mayor calado asociados a las alteraciones de la accesibilidad y de la funcionalidad del territorio articulado por la carretera.

La metodología diseñada aporta, como aspecto a reseñar, por su novedad y complejidad, su perspectiva integradora, en diversos sentidos. 
Figura 10. Estructura de la interfaz informática

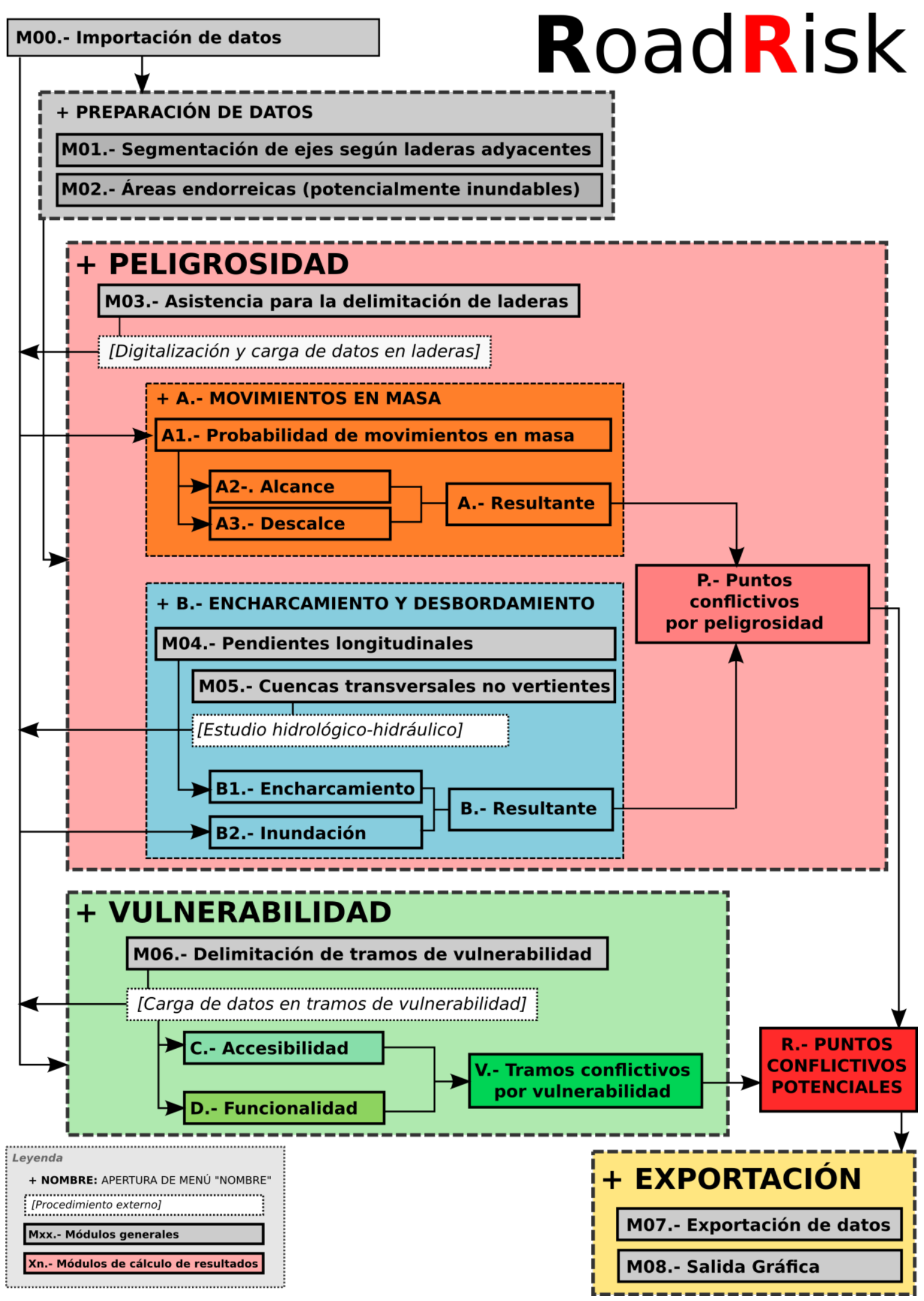

Fuente: elaboración propia 


\section{Figura 11. Perspectiva transversal integrada}

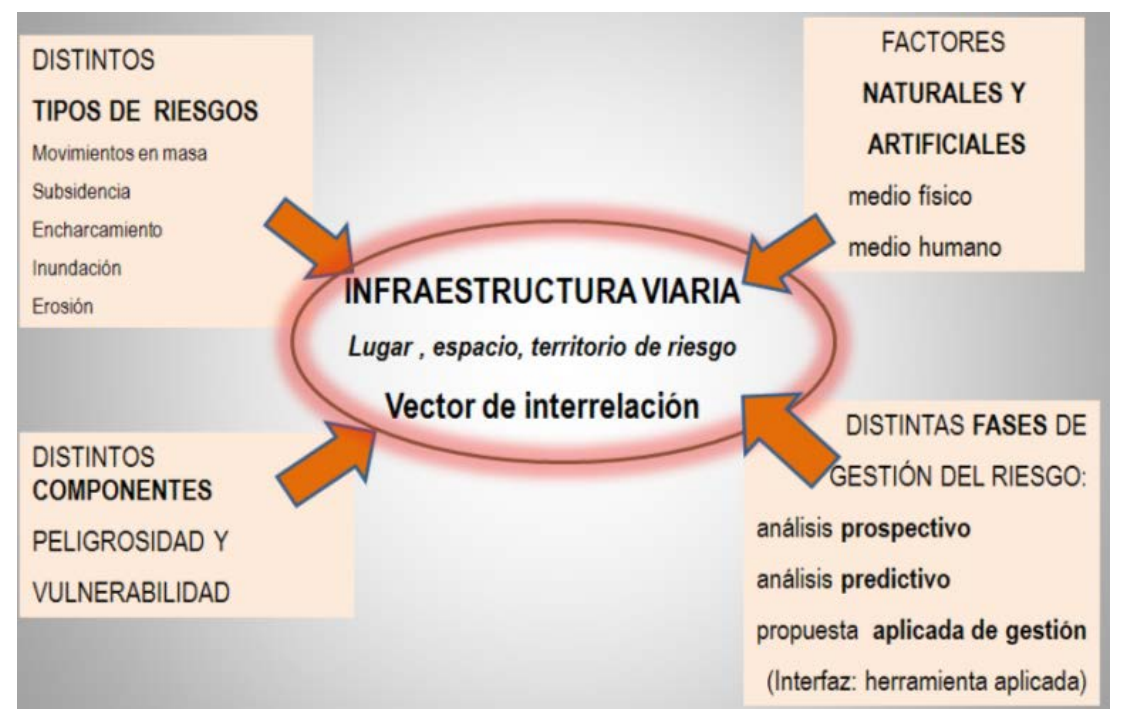

Fuente: elaboración propia

Se han tratado de forma unitaria e interrelacionada distintos tipos de peligros naturales, valorando sus posibles efectos sinérgicos. Se han interrelacionado igualmente factores procedentes tanto del medio natural como artificial, adaptando en cada punto la metodología de análisis y estimación a las particularidades de naturaleza y funcionamiento de los distintos factores implicados. Se ha integrado, de forma efectiva, la cartografía de peligrosidad junto a la de vulnerabilidad, para orientar los resultados hacía las carreteras que producirán impactos más trascendentes en el funcionamiento de los servicios territoriales. Los datos indicadores de los distintos riesgos y sus consecuencias sobre el funcionamiento del viario se entrelazan en distintos puntos de la metodología, con el objetivo finalista de producir resultados veraces, afines en su complejidad al funcionamiento real de estos procesos en situaciones de crisis, cuando todos los factores interactúan en el tiempo y en el espacio de la infraestructura viaria. En resumen, en el estudio se ha aplicado un tratamiento transversal de información múltiple y compleja, y de se ha tratado de forma interconectada problemas y procesos de procedencia muy diversa, con objeto de fin de orientar la investigación de base hacia una finalidad aplicada.

Otro de los logros del trabajo ha sido la generación de una interfaz que permite la aplicación ágil de esta metodología, a priori, compleja, a otras zonas de estudio. La aplicación informática diseñada puede ser útil para la adopción de medidas preventivas en infraestructuras ya construidas, y también emplearse en la fase de proyecto para simular cuál sería el grado de peligro en diferentes alternativas de trazados planteadas. Ofrece, asimismo, criterios para diseñar y reforzar la señalización de la zona, de utilidad en la fase de gestión del tráfico, especialmente en la fase crítica del episodio torrencial. La elaboración y consulta de este tipo de cartografía permite a los gestores del mantenimiento de la infraestructura diferenciar de forma simultánea la ubicación, tipología y causas del punto de peligrosidad, y, a su vez, localizar de forma precisa qué tramo de carretera 
está afectando y que vulnerabilidad tiene el mismo. De este modo se genera un documento sintético, con la información necesaria para priorizar actuaciones o medidas preventiva según lo crea oportuno. Los resultados del estudio presentan un elevado interés para la mejora de la seguridad y la funcionalidad del viario en situaciones críticas. Las utilidades de la aplicación son múltiples, y los beneficiarios se sitúan tanto en el ámbito de la consultoría privada, como en el de la gestión pública de las carreteras.

Por todos estos rasgos, puede concluirse que, tanto la metodología como la aplicación informática que la implementa, constituyen una aportación innovadora para la prevención del riesgo y de la gestión de la catástrofe en el ámbito de las infraestructuras viarias.

Agradecimientos: Los contenidos del artículo se fundamentan en los trabajos realizados en el marco de un Proyecto de Investigación financiado por la Agencia de la Obra Pública y la Consejería de Infraestructuras y Vivienda de la Junta de Andalucía (GGI3002IDIS).

Declaración responsable: Las/os autoras/es declaran que no existe ningún conflicto de interés en relación con la publicación de este artículo. M.J. Perles Roselló: Conceptuación teórica y elaboración de la propuesta metodológica conjunta. Calibración estadística de los resultados. S. Pardo García: Aplicación de la metodología al caso de estudio y diseño técnico de la interfaz informática. M. Mérida Rodriguez: Diseño de la metodología y cartografía correspondiente al Bloque II (Vulnerabilidad), y coordinación de la aplicación de la misma. J. Olcina Cantos: Elaboración de antecedentes y estado de la cuestión y supervisión de procedimientos. 


\section{Bibliografía}

Ardizzone, E., Cardinali, M., Carrara, A., Guzzetti, F., \& Reichenbach, P. (2002). Impact of mapping errors on the reliability of landslide hazard maps. Natural Hazards and Earth System Sciences, 2, 3-14.

Ataollah, S., Lee, S., Oh, H., \& Kamran, C. (2012). A GIS-based logistic regression model in rock fall susceptibility mapping along a mountainous road: Salavat Abad case study, Kurdistan, Iran. Natural Hazards, 64, 1639-1656. http://dx.doi.org/10.1007/s11069-012-0321-3

Ayalew, L., \& Yamagishi, H. (2005). The application of GIS-based logistic regression for landslide susceptibility mapping in the Kakuda-Yahiko Mountains, Central Japan. Geomorphology, 65, 1531.

Barredo, J. I., Benavides, A., Hervás, J., \& Van Westen, C. J. (2000). Comparing heuristic landslide hazard assessment tecniques using GIS in the Tirajana basin, Gran Canaria island, Spain. International Journal of Applied Earth Observation and Geoinformation, 2(1), 923. https://doi.org/10.1016/S0303-2434(00)85022-9

Blaikie, P., Cannon, T., Davis, I., \& Wisner, B. (1994). At Risks: Natural Hazards, People Vulnerability and Disasters. Routledge.

Bornaetxea, T., Ormaetxea, O., \& Antigüedad, I. (2016). Modelo de susceptibilidad de deslizamientos superficiales para la cuenca del río Oria (Guipuzcoa). Aplicación de la regresión logística y comparación de resultados con estudios precedentes. In J. J. Durán, M. Montes, A. Robador \& A. Salazar (Eds.), Comprendiendo el relieve: del pasado al futuro. Actas de la XIV Reunión Nacional de Geomorfología. Madrid.

Brice, J. C. (2001) [1984]. Assessment of Channel Stability at Bridge Sites. Transportation Research Record, Vol. 2, No. 950, Transportation Research Board, Washington, D.C. 20418. In F. Calvo García-Tornell, Sociedades y territorios en riesgo. Barcelona: Ediciones del Serbal.

Calvo García-Tornell, F. (2001). Sociedades y territorios en riesgo. Barcelona: Ediciones del Serbal.

Camarasa Belmonte, A. M, \& Soriano García, J. (2008). Peligro, vulnerabilidad y riesgo de inundación en ramblas mediterráneas. Los llanos de Carraixet y Poyo. Cuadernos de Geografía, $83,1-26$.

Carrasco, R. M., Pedraza, J., Martín Duque, J. F., Mattera, M., Sanz M. A., Bodoque, J. M., \& Diez Herrero, A. (2003). Cartografía de zonas de susceptibilidad o propensión a los movimientos en masa en el Valle del Jerte (Sistema Central Español). In F. J. Ayala-Carcedo \& J. Corominas (Ed.). 
Mapas de susceptibilidad a los movimientos de ladera con técnicas SIG. Fundamentos y Aplicaciones en España (pp. 133-153). Madrid: Publicaciones del Instituto Geológico y Minero de España.

Casale, R., \& Margotinni, C. (1999). Flood and landslides: Integrated Risk Assesment. Germany: Ed. Springer Science \& Bussines Media.

Chacón, J., Irigaray, C., Fernández del Castillo, T., \& El Hamdouni, R. (2003). Susceptibilidad a los movimientos de ladera en el sector central de la Cordillera Bética. In F.J. Ayala Carcedo \& J. Corominas, (Eds.), Mapas de susceptibilidad a los movimientos de ladera con técnicas SIG. Fundamentos y Aplicaciones en España (pp. 83-96). Madrid: Publicaciones del Instituto Geológico y Minero de España.

Chau K., Tang Y., \& Wong R. (2004). GIS-Based Rock-fall susceptibility map for Hong Kong. International Journal of Rock Mechanics and Mining Sciences, 41(3), 530-542.

Clark, G. E., Moser, S. C., Ratick, S. J., Dow, K., Meyer, W. B., Emani, S., ... Scharz, H. E. (1998). Assessing the vulnerability of coastal communities to extreme storms: the case of Revere, $M$ A, USA. Mitigation and Adaptation Strategies for Global Change, 3(1), 5982. https://doi.org/10.1023/A: 1009609710795

Conesa-García, C., García-Lorenzo, R., \& Pérez-Cutillas, P. (2017). Flood hazards at ford stream crossings on ephemeral channels (south-east coast of Spain). Hydrological Processes, 31, 731749. https://doi.org/10.1002/hyp. 11082

Conesa García, C., \& García Lorenzo, R. (2014). Flood hazard assessment for bridge crossings over ephemeral channels: A case study of the Murcia Coast (SE Spain). Cuadernos de Investigación Geográfica, 40(1), 117143. https://doi.org/10.18172/cig.2507

Conesa García, C., \& García Lorenzo, R. (2013). Evaluating the effectiveness of road-crossing drainage culverts in ephemeral streams. Hydrological Processes, 27(12), 17811796. https://doi.org/10.1002/hyp.9335

Corominas, J., Ibarbia, I., Luzuriaga, S., Navarro, J. A., Jujo, I., Jurnet, C., \& Hurlimann, M. (2013). Rockfall and debris flow hazard assesment of the coastal road of Guipuzkoa (Northern Spain). In C. Margottini, P. Canuti, K. Sassa (Eds.), Landslides Science and Practice, vol. 6, Risk Assesment, Management and Mitigation. Germany: Springer.

Cutter, S., Emrich, C., Gale, M., \& Reeves, R. (2018). Flash Flood Risk and the Paradox of Urban Development. Natural Hazards Review, 19(1). https://doi.org/10.1061/(ASCE)NH.1527$\underline{6996.0000268}$ 
Cutter, S.; Boruff, B., \& Shirley, W. (2003). Social vulnerability to environmental hazards. Social Science Quarterly, 84(2), 242-261.

Cutter, S. (1994). Environmental Risk and Hazards. Englewood Cliffs, Prentice-Hall.

Delmonaco G., Garbin F., Marsella M., Margottini C., Sonnessa A., \& Spizzichino D. (2013). Laser Scanning Analysis and Landslide Risk Assessment on Transportation Network: The Lugnano in Teverina (Umbria Region, Italy), Landslide Case Study. In C. Margottini, P. Canuti, K. Sassa (Eds.), Landslide Science and Practice. Berlin: Springer.

Delmonaco, G., Margottini, C., \& Spizzino, D. (2006). Report on new methodology for multi-risk assesment and the harmonisation fo different natural risk maps. ARMONÍA Proyect: Applied multirisk mapping of Natural Hazards for Impact Assessment. Deliverable 3. 1. European Community.

Diez Herrero, A., Laín Huerta, L., \& Llorente Isidro, M. (2008). Mapas de peligrosidad por avenidas e inundaciones. Guía metodológica para su elaboración. Madrid: Publicaciones del IGME.

Felicísimo, A., Cuartero, A., Remondo, R., \& Quirós, E. (2013). Mapping landslide susceptibility with logistic regression, multiple adaptive regression splines, classification and regression trees, and maximum entropy methods: a comparative study. Landslides, 10(2), 175-189.

Fell, R., Corominas, J., Bonnard, C., Cascini, L., Leroi, E., \& Savage, W. Z. (2008). Guidelines for landslide susceptibility, hazard and risk zoning for land-use planning. Engineering Geology, 102, 99-111. https://doi.org/10.1016/j.enggeo.2008.03.022

Furniss, M. J., Ledwith, T. S., Love, M. A., McFadin, B. C., \& Flanagan, S. A. (1998). Response of Road-stream Crossings to Large Flood Events in Washington, Oregon, and Northern California. United States Department of Agriculture. Forest Service - San Dimas Technology and Development Center.

González-Jiménez, R. M., Carrasco, F. J., Ayala Carcedo, Pedraza Gilsanz, J., Martín-Duque, J. F., Sanz, M. A., \& Bodoque, J. M. (2007). El análisis de la susceptibilidad en la prevención de los movimientos de ladera: un análisis comparativo de las metodologías aplicadas al Valle del Jerte (Sistema Central español). In F.J. Ayala Carcedo, J. Olcina Cantos, L. Laín Huerta \& A. González Jiménez (Eds.), Riesgos naturales y desarrollo sostenible. Impacto, predicción y mitigación (pp. 221-246). Madrid: IGME.

Guzzetti, F., Carrara, A., Cardinali, M., \& Reichenbach, P. (1999). Landslide hazard evaluation: a review of current techniques and their application in a multi-scale study, Central Italy. Geomorphology, 31, 181-216 
Greiving, S., Fleischhauer, M., \& Lückenkötter, J. (2004). Dealing with hazards: Multi risk mapping of Europe's regions and its policy implications. Grenoble, France: AESOP.

Hosmer Jr, D. W., \& Lemeshow, S. (2004). Applied logistic regression. Oxford: John Wiley \& Sons.

Ikeya, H. (1989). Debris flow and its countermeasures in Japan. Bulletin of the International Association of Engineering Geology, 40(1), 15-33.

Jones, J. A., Swanson, F. J., Wemple, B. C., \& Snyder, K. U. (2000). Effects of roads on hydrology, geomorphology, and disturbance patches in stream networks. Conservation Biology, 14(1), 76-85.

Lagasse, P. F., Schall, J. D., \& Richarson, E. V. (2012). Stream Stability at Highway Structures. Hydraulic Engineering Circular 20, Publication No. FHWA-HIF-12-004. Federal Highway Administration, U.S. Department of Transportation, Washington DC. Retrieved from https://www. fhwa.dot.gov/engineering/hydraulics/pubs/hif12004.pdf

Lexer, W., Paluchova, K., \& Schwarzl, B. (2006). Risk Assessment. IMProving the IMPlementation of Environmental IMPact Assessment, (IMP)3. Risk Assessment D 3.2 Report WP 3. Vienna: Österreichisches Institut für Raumplanung.

Llorente Isidro, M., Díez Herrero, A., \& Laín Huerta, L. (2006). La experiencia del IGME en cartografía de peligrosidad de avenidas torrenciales e inundaciones: de Casiano de Prado a PRIGEO. In A. Díez Herreros, L. Laín Huerta, \& M. Llorente Isidro, M. (Eds), Mapas de peligrosidad de avenidas e inundaciones. Métodos, experiencias y aplicación (pp. 41-63). Madrid: Publicaciones del Instituto Geológico y Minero de España.

Luce, C. H., \& Wemple, B.C. (2001). Introduction to special issue on hydrologic and geomorphic effects of forest roads. Earth Surface Processes and Landforms, 26, 111-113.

Máyer Suárez, P. (2002). Desarrollo urbano e inundaciones en la ciudad de Las Palmas de Gran Canaria (1869-2000). Investigaciones geográficas, 28, 145-159.

Mérida Rodriguez, M., Perles Roselló, M.J., \& Blanco Sepúlveda, R. (1998). Urbanización, infraestructuras y riesgos naturales en la periferia montañosa de la ciudad de Málaga. El caso del monte San Antón. Revista Baética, 20,129157. http://dx.doi.org/10.24310/BAETICA.1998.v0i20.511

Montz, B. E. (1994). Methodologies for analysis of multiple hazard probabilities: An application in Rotura, New Zealand. Centre for Environmental and Resource Studies, University of Waikato, Hamilton. 
Nilsen, M. W. (2008). Modelling of rockfall runout range: employing empirical and dinamical methods (Master Thesis, Universitetet i Oslo, Norway). Retrieved from http://urn.nb.no/URN:NBN:no-19773

Olcina Cantos, J. (2008). Cambios en la consideración territorial, conceptual y de método de los riesgos naturales. In X Coloquio Internacional de Geocrítica, Diez años de cambios en el mundo, en la Geografía y en las ciencias sociales, 1999-2008. Barcelona, 26 - 30 de mayo de 2008. Retrieved from http://www.ub.es/geocrit/-xcol/62.htm

Olcina Cantos, J., \& Ayala Carcedo, F. J. (2002). Riesgos naturales. Madrid: Ariel.

Olcina Cantos, J., \& Diez Herrero, A. (2017). Cartografía de inundaciones en España. Revista Estudios Geográficos, 78(282), 283-315.

Olcina Cantos, J., Sauri, D., Hernández, M., \& Ribas, A. (2016). Flood policy in Spain: a review for the period 1983-2013. Disaster Prevention and Management: an International Journal, 25(1), $41-58$.

Ollero Ojeda, A. (2014). Guía metodológica sobre buenas prácticas en gestión de inundaciones (Manual para gestores). Contrato del río Matarraña. Zaragoza: Fundación Ecología y Desarrollo. Retrieved from:

http://contratoderiomatarranya.org/documentos/Guia_BB_Gestion_inundaciones.pdf

Pedraza, J., Carrasco, R. M., Bodoque, J. M., Sanz, M. A., Martín-Duque, J. F., González, A., \& Díez, A. (2004). The Jubaguerra stream event: analysis of a mass movement connected with a flash flood phenomenon and its application to other areas in the Gredos Mountains (Central Spain). In C. A. Brebbia (Ed.), Risk Analysis IV (pp. 345-358). Southampton, United Kingdom: Wessex Institute of Technology (WIT Press).

Perles Roselló, M.J., \& Cantarero Prados, F. (2010). Problemas y retos en el análisis de los riesgos múltiples del territorio: propuestas metodológicas para la elaboración de cartografías multi-peligros. Boletín de la Asociación de Geógrafos Españoles, 52, 245-271. Retrieved from https://www.age-geografia.es/ojs/index.php/bage/article/view/1171

Perles Roselló, M. J., \& Mérida Rodriguez, M. F. (2010). Patrón territorial y conformación del riesgo en espacios periurbanos. El caso de la periferia este de la ciudad de Málaga. Revista Scripta Nova, XIV. http://dx.doi.org/10.1344/sn2010.14.1635

Perles Roselló, M. J., Vías Martínez, J., \& Andreo Navarro, B. (2008). Vulnerability of human environment to risk: case of groundwater contamination risk: Environment International. https://doi.org/10.1016/j.envint.2008.08.005 
Perles, M. J., Cabello, J., López, C., Vallejo, J. A., \& Vías, J. M. (1999). El problema de la inundación/ocupación en el Bajo Guadalhorce, efectos de la ocupación humana de un área inundable. Jábega, 81.

Petrucci, O., \& Pasqua, A. A. (2012). Damaging events along roads during bad weather periods: a case study in Calabria (Italy). Natural Hazards and Earth System Sciences, 12, 365-378.

Razavizadeh, S., Solaimani, K., Massironi, M., \& Kavian, A. (2017). Mapping landslide susceptibility with frequency ratio, statistical index, and weights of evidence models: a case study in northern Iran. Environmental Earth Sciences, 76, 499. https://doi.org/10.1007/s12665-017$\underline{6839-7}$

Remondo, J., Soto, J., González-Díez, A., Díaz de Terán, J. R., \& Cendrero, A. (2005). Human impact on geomorphic processes and hazards in mountain areas in northern Spain. Geomorphology, 66, 69-84. https://doi.org/10.1016/j.geomorph.2004.09.009

Rickenmann, D. (1999). Empirical relationships for debris flows. Natural hazards, 19(1), 47-77.

Rsavdaroglou, M., Al-jibaouri, H., Bles, T., \& Halman, J. (2018). Proposed methodology for risk analysis of interdependent critical infrastructures to extreme weather events. International Journal of Critical Infrastructure Protection, 21, 57-71.

San Millan, E., González-Díez, A., \& Fernández-Maroto, G. (2016). Influencia de las precipitaciones en los movimientos de ladera en Cantabria. In J. J. Durán, M. Montes, A. Robador, \& A. Salazar, A. (Eds.), Comprendiendo el relieve: del pasado al futuro (pp. 265-272). Madrid: Actas de la XIV Reunión Nacional de Geomorfología.

Sauri Pujol, D. (2004). Tendencias recientes en el análisis geográfico de los riesgos ambientales. Áreas, 23. Retrieved from http://revistas.um.es/areas/article/view/117861

Saurí Pujol, D., \& Ribas Palom, A. (1994). El análisis del riesgo de avenida en las escuelas geográficas anglosajona, francesa y española. Estudios Geográficos, 216, 481-502.

Serck, L. (2012, April 30). Dangers of river ford crossings. In BBC News Online South. Retrieved from http://www.bbc.com/news/uk-england-hampshire-17897637.

Simon, A., \& Downs, P. (1995). An interdisciplinary approach to evaluation of potential instability in alluvial channels Geomorphology, 12(3), 215-232. https://doi.org/10.1016/0169$\underline{555 \times(95) 00005-P}$

Sortino Barrionuevo, J.F., Mérida Rodriguez, M. F., \& Perles Roselló, M. J. (2016). Susceptibilidad de movimientos en masa en infrestructuras viarias. Aplicación a un tramo de la autovía A-7 (circunvalación de Málaga). In J. J. Durán, M. Montes, A. Robador, \& A. Salazar (Eds.), 
Comprendiendo el relieve: del pasado al futuro (pp. 273-282). Madrid: Actas de la XIV Reunión Nacional de Geomorfología.

Tibaldi, A., Ferrari, L., \& Pasquare, G. (1995). Landslides triggeres by earthquakese and their relations with faults and mountain slope geometry: an example from Ecuador. Geomorphology, $17(3), 215-226$.

Varnes, D. J. (1978). Slope movement types and processes. In R. L. Schuster \& R. J. Krizek (Eds.), Special Report 176: Landslides: Analysis and Control. Transportation and Road Research Board (pp. 11-33). Washington D. C.: National Academy of Science. Retrieved from http://onlinepubs.trb.org/Onlinepubs/sr/sr176/176-002.pdf

Veyret, Y., Beucher, S., \& Bonnard, Y. (2005). Risques naturels et territoires Bulletin de I'Association de géographes français, 2005(1), 63-74.

Wei Chen, H., \& Zhou, Z. (2016). A GIS-based comparative study of DempsterShafer, logistic regression and artificial neural network models for landslide susceptibility mapping. Geocarto International, 32(4), 367-385. https://doi.org/10.1080/10106049.2016.1140824

Yilmaz, I. (2009). Landslide susceptibility mapping using frequency ratio, logistic regression, artificial neural networks and their comparison: a case study from Kat landslides (Tokat-Turkey). Computers and Geosciences, 35, 1125-1138.

Youssef, A.M., Pradhan, B., \& Hassan, A. M. (2011). Flash flood risk estimation along the St. Katherine road, southern Sinai, Egypt using GIS based morphometry and satellite imagery. Environmental Earth Sciences, 62(3), 611-623.

Zhang, S., Zhang, L. M., Chen, H. X., Yuan, Q., \& Pan, H. (2013). Changes in runout distances of debris flows over time in the Wenchuan earthquake zone. Journal of Mountain Science, 10(2), 281-292.

Zhao, C., Lin, N., \& Fang, D. (2018). Criticality assessment of urban interdependent lifeline systems using a biased PageRank algorithm and a multilayer weighted directed network model. International Journal of Critical Infrastructure Protection, 22, 100112. https://doi.org/10.1016/j.ijcip.2018.06.002 